\title{
Revision of Defeasible Logic Preferences
}

\author{
Guido Governatori ${ }^{\bullet}$, Francesco Olivieri ${ }^{\bullet \bullet}$, \\ Simone Scannapieco $^{\dagger \bullet *}$ and Matteo Cristani ${ }^{\dagger}$ \\ ${ }^{\dagger}$ Department of Computer Science, University of Verona, Italy \\ -NICTA, Queensland Research Laboratory, Australia \\ *Institute for Integrated and Intelligent Systems, Griffith University, Australia
}

\begin{abstract}
There are several contexts of non-monotonic reasoning where a priority between rules is established whose purpose is preventing conflicts.

One formalism that has been widely employed for non-monotonic reasoning is the sceptical one known as Defeasible Logic. In Defeasible Logic the tool used for conflict resolution is a preference relation between rules, that establishes the priority among them.

In this paper we investigate how to modify such a preference relation in a defeasible logic theory to change the conclusions of the theory itself. We argue that the approach we adopt is applicable to legal reasoning, where users, in general, cannot change facts or rules, but can propose their preferences about the relative strength of the rules.

We provide a comprehensive study of the possible combinatorial cases and we identify and analyse the cases where the revision process is successful.

After this analysis, we identify three revision/update operators and study them against the AGM postulates for belief revision operators, to discover that only a part of these postulates are satisfied by the three operators.
\end{abstract}

\section{Introduction}

A large number of real-life cases in legal reasoning, argumentation theory, information security, digital forensics, and even engineering or medical diagnosis, exhibit two common traits: (a) different persons have different preferences, and (b) it can be the case that one conclusion taken with a given preferential order is not taken with a different order. When the decision mechanism is based on rules and such rules are in conflict, inconsistencies may be generated and decision making may require some (preference) mechanism to solve/avoid conflicts. Unfortunately, it may occur that using a particular set of preferences to solve a conflict does not result in the desired/expected outcome. That being the case, to change the outcome, we need to revise the underlying preferences.

More specifically, non-monotonic reasoning has been advanced for common-sense reasoning as well as reasoning with partial and conflicting information. We can distinguish two types of non-monotonic reasoning: credulous and sceptical. In credulous nonmonotonic reasoning, once a conflict arises, we independently explore the two branches of the conflict. On the contrary, sceptical non-monotonic formalisms provide means to solve conflicts. In this paper, we concentrate on sceptical non-monotonic reasoning. 
In fact, a credulous framework would simply accommodate the conflict by generating conflicting branches with no need to solve the conflict by specifying preferences.

Sceptical non-monotonic formalisms are typically equipped with techniques to address conflicts, where a conflict is a combination of reasoning chains leading to a contradiction (such as opposite conclusions). The most common device to handle conflicts is a preference or superiority relation over the elements used by the formalism to reason. These elements can be formulae, axioms, rules or arguments, and the preference relation states that one of such elements is to be preferred to another one when both can be used. Preference/superiority relation-based mechanisms to solve conflicts have been extensively used by scholars in many areas of AI; for a recent survey see [1].

In this research, we focus on a specific rule-based non-monotonic formalism, that of Defeasible Logic, but the motivation behind the particular technical development applies in general to other rule-based non-monotonic formalisms equipped with a priority among rules (or other elements). Indeed, when considering a rule-based formalism, knowledge is typically partitioned into facts (describing immutable propositions/statements about a case), rules (describing relationships between the atoms of the language), and a preference or superiority relation (describing the relative strength of rules). A revision operation ${ }^{1}$ transforms a theory by changing some of its elements, be it the facts, the rules, or the superiority relation. A revision based on changing the facts corresponds to an update operation [2], revision based on modification of rules has been investigated in [3], whilst to the best of our knowledge, revision of non-monotonic theories based on modifications of the underlying superiority relation has been neglected so far.

The focus of this work is to study the revision of sceptical, non-monotonic theories by operating only on the superiority relation. We begin by arguing that this form of theorychanging has natural correspondences to reasoning patterns in legal reasoning, like the situation when a person/lawyer who is disputing a case can neither contest the evidences of the case, nor change the legal system, but they can argue about which norms apply to which situation. We shall prove that the problem of deciding whether a theory can be revised by changing only the superiority relation is NP-complete. Lastly, we shall conduct a thorough investigation of whether the AGM belief revision postulates are appropriate for a non-monotonic setting.

The AGM postulates were designed with classical logic in mind. Classical logic is monotonic. As such, if we add new information which is incompatible with the old one, an inconsistency arises. In this scenario, the sole way to recover consistency is to invoke a revision operator.

Conversely, due to the nature of non-monotonic reasoning, adding new "incompatible" information in a non-monotonic system typically does not generate a contradiction within the theory, even if the result may not be conceptually satisfactory.

Given the difference in nature between classical logic and non-monotonic reasoning, it is of interest to investigate which of the AGM postulates apply to non-monotonic reasoning, to what extent, and in which form.

\footnotetext{
${ }^{1}$ In general, we shall use the term revision operation to denote any operation that changes a theory. In Sections 4 and 7 the term will be understood in a specific technical sense.
} 
In the recent years, a few works addressed the issue of belief revision in nonmonotonic settings where there is a general understanding that the AGM postulates are not fully appropriate for non-monotonic reasoning. For instance, [4] shows that belief revision methodologies are not suitable to changes in specific significant nonmonotonic theories, and that it is possible to revise such theories fully satisfying the AGM postulates, but then the outcome is utterly meaningless for their purposes.

Still, the matter whether and which postulates hold is far from being settled. For example, [5] proposed an approach to belief revision of logic programs under answer set semantics that is fully compliant with the base AGM postulates for revision. On the other hand, Delgrande [6, p. 568] asserts that a subset of postulates for belief revision is not appropriate for belief revision of non-monotonic theories (and thus is ignored in his work). At the same time some other work (e.g., [7]) attempts to reformulate AGM postulate to fit (some) non-monotonic formalisms. This suggests that the suitability of AGM postulates to a belief revision approach for non-monotonic reasoning is still debatable.

Our agenda is as follows. In Section 2, we motivate that reasoning over preferences on rules and on how to modify the preferences is a natural reasoning pattern in legal reasoning. We then introduce, in Section 3, Defeasible Logic, the formalism chosen for our investigation. Section 4 is dedicated to prove that the problem of revising a theory by only changing the superiority relation is an NP-complete problem; in Section 5 we briefly motivate the types of changes we study by appealing to the legal domain. Section 7 analyses the AGM postulates for revision of defeasible preferences. Section 8 overviews closely related approaches, and Section 9 concludes the paper with a summary of the achieved results, discussion of related works and quick hints for future developments.

\section{Norms and Preferences in Legal Reasoning}

The main intuition in such paradigms is that norms can be represented by rules, the evidence in cases by facts, and that the superiority relation is induced by legal principles determining how to solve conflicts between norms. Similarly, in argumentation, given two conflicting arguments, the superiority relation establishes which argument is stronger than the other $[8,9]$.

We take the stance that, particularly in the legal reasoning domain, we do not have control over the rules (norms), or their modification, but have some control on how they can be used. In fact, an average citizen has no power to change the law, and has no power on what norms are effective in the jurisdiction they are situated in. These powers are instead reserved to persons, entities and institutions specifically designated to do so, for example the parliament and, under some given constraints, also the judges - in Common Law juridical systems, especially.

A citizen can however argue that one norm instead of another applies in a specific case. This amounts to saying that one norm is to be preferred to the other in that case.

Prima-facie conflicts appear in legal systems for a few main reasons, among which we can easily identify three major representatives: (1) norms from different sources, (2) norms promulgated at different times, and (3) exceptions. These phenomena are well 
understood and have given rise to principles, which existed for a long time in legal theory and been used to solve such issues. These principles are used in many situations, such as an argument to drive constitutional judgement against a given norm or a given sentence. Here we list the three major legal principles, expressing preferences among rules to be applied [10].

Lex Superior This principle states that when there is a conflict between two norms from different sources, the norms originating from the legislative source higher in the legislative source hierarchy takes precedence over the other norm. This means that if there is a conflict between a federal law and a state law, the federal law prevails over the state law.

Lex Posterior According to this principle, a norm promulgated after another norm takes precedence over the older norm.

Lex Specialis This principle specifies that when a norm applies to a specific set of admissible circumstances, and another norms applies under more general conditions, the most specific norm prevails.

Besides the above principles, a legislator can explicitly establish that one norm prevails over another conflicting norm. The intuition behind these principles is that when there are two conflicting norms, and the two norms are both applicable in a specific case, we can apply one of these principles to create an instance of the superiority relation that discriminates between the two conflicting norms. However, there are further complications. What if several principles may apply at the same time and these produce opposite preferences? Do the preferences lead to opposite outcomes of a case? These are examples of situations when revision of preferences is relevant. The following example illustrates this situation.

Example 1. Charlie is an immigrant son of an Italian. Living in Italy, he is interested in joining the Italian Army, based on Law 91 of 1992. However, his application is rejected, based upon a constitutional norm (Article 51 of the Italian Constitution allowing Italian citizens to be eligible for public services). The two norms Law 91 and Article 51 are in conflict, thus the Army's decision is based on the lex superior principle. Charlie appeals against the decision in court. The facts of the case are undisputed, and so are the norms to be applied and their interpretation. The only chance for Bob, Charlie's lawyer, to overturn the decision is to argue that Law 91 overrides Article 51 of the Constitution. Thus, Bob counter-argues by appealing to the lex specialis principle since Law 91 of 1992 explicitly covers the case of a foreigner who applies for joining the Army with the purpose of obtaining the Italian citizenship.

The two arguments do not address the facts and rules that hold in the case. They disagree about which rule prevails over the other, Article 51 of the Constitution or Law 91. In particular, Bob's argument can be seen as an argument where the relative strength of the two rules is reversed compared to the argument of the Army's lawyer, and it can be used to revise the previous decision.

The mechanism outlined above relates to the notion of strategical reasoning, where a discussant looks at the best argument to prove a given claim (in this case, one set of rules prevail over another). However, the key aspect is that, before undertaking the 
process of understanding which argument is the most likely to win the argument, one has to ensure that changing the superiorities actually leads to a different outcome of the claim (and possibly the desired result).

It is not our aim to study how to support a given set of preferences using argumentation ${ }^{2}$. In this work, we investigate whether it is possible to modify the consequences of a theory (as represented by a defeasible theory) only through changes on the superiority (preference) relation. We hence believe that our framework is foundational for arguments about preferences. This means that one can first determine whether the outcome of a discussion can be turned in her favour by only changing the superiority relation, and then figure out which argument supports the preference.

In the current literature about formalisms apt to model normative and legal reasoning, a simple and efficient non-monotonic formalism which has been discussed in the community is Defeasible Logic. This system is described in detail in the next section.

One of the strong aspects of Defeasible Logic is its characterisation in terms of argumentation semantics [12]. In other words, it is possible to relate it to general reasoning structure in non-monotonic reasoning which is based on the notion of an admissible reasoning chain. (An admissible reasoning chain is an argument in favour of a thesis/claim.) For these reasons, much research effort has been spent upon Defeasible Logic since it encompasses some other (sceptical) formalisms proposed for legal reasoning $[12,13,14,15]$.

Most interestingly, in Defeasible Logic we can reach positive conclusions as well as negative conclusions. It thus gives us understanding to both accepting a conclusion as well as rejecting a conclusion. This is particularly advantageous when trying to address the issues determined by reasoning conflicts, and has a strong applicability in legal reasoning.

\section{Defeasible Logic}

We divided the present section in three subsections. We start by presenting standard notions in Defeasible Logics, its language, atoms, and how its proof theory works, including its standard proof tags. The accustomed reader will find standard concepts in Section 3.1. We then introduce two new proof tags, whose use will be essential to prove formal properties in the rest of the paper (Section 3.2). We conclude with Section 3.3, where we advance some properties of the logic and preliminary results, and we propose a real-life example, which is formalised into our logic.

\subsection{Language and notation}

A defeasible theory $D$ is a tuple $D=(F, R,>)$ and consists of five different kinds of knowledge. (Examples of facts and rules below are standard in the literature of the field.)

Set $F$ denotes the facts of the theory, simple pieces of information that are considered always to be true. For example, a fact is that "Sylvester is a cat", formally

\footnotetext{
${ }^{2}$ A similar problem, that of deciding which is the best set of rules to put forward in a two-players dialogue game, was studied in [11], where the authors proved that the problem is NP-complete.
} 
cat (Sylvester). $R$ is the set of rules and comprehends: strict rules, defeasible rules, and defeaters. A rule ' $r: A(r) \hookrightarrow C(r)$ ' consists of (i) its antecedent $A(r)$ (or body), which is a finite set of literals, (ii) the arrow $\hookrightarrow \in \rightarrow, \Rightarrow, \sim(\rightarrow$ for strict rules, $\Rightarrow$ for defeasible rules, and $\sim$ for defeaters), and (iii) its consequent (or head) $C(r)$, which is a single literal.

A strict rule is a rule in which, whenever the premises are indisputable (e.g., facts), so is the conclusion. For instance,

$$
\operatorname{cat}(X) \rightarrow \operatorname{mammal}(X)
$$

means that "every cat is a mammal".

A defeasible rule is a rule that can be defeated by contrary evidence; for example, "cats typically eat birds" can be represented by

$$
\operatorname{cat}(X) \Rightarrow \operatorname{eatBirds}(X) .
$$

The underlying idea is that if we know that something is a cat, then we may conclude that it eats birds, unless there is evidence proving otherwise. Defeaters, finally, are special types of rules that cannot be used to draw any conclusion. Their only use is to prevent some conclusions by producing evidence to the contrary. An example is "if a cat has just fed itself, then it might not eat birds":

$$
\text { justFed }(X) \leadsto \neg \text { eatBirds }(X) .
$$

The set of all strict rules in $R$ is denoted by $R_{s}$, while the set of strict and defeasible rules by $R_{s d}$. We name $R[q]$ the rule set in $R$ with head $q$.

Lastly, the superiority relation $>$ is a binary relationship over the set of rules defining when one rule overrides the conclusion of another rule. For instance, given the defeasible rules

$$
\begin{aligned}
r_{0}: \operatorname{cat}(X) & \Rightarrow \operatorname{eat} \operatorname{Birds}(X) \\
r_{1}: \operatorname{domesticCat}(X) & \Rightarrow \neg \text { eatBirds }(X)
\end{aligned}
$$

which would contradict one another when Sylvester is both a cat and a domestic cat, they do not in fact contradict if we state that $r_{1}>r_{0}\left(r_{1}\right.$ is stronger than $\left.r_{0}\right)$, leading Sylvester not to eat birds. In Defeasible Logic, as in many different non-monotonic formalisms, the superiority relation determines the relative strength of two conflicting rules, i.e., rules whose heads are complementary. The complementary of a literal $q$ is denoted by $\sim q$; if $q$ is a positive literal $p$, then $\sim q$ is $\neg p$, and if $q$ is a negative literal $\neg p$ then $\sim q$ is $p$.

Like in [16], we consider only a propositional version of this logic, and we do not take into account function symbols. Every expression with variables represents the finite set of its variable-free instances.

At the heart of the reasoning mechanism of the logic is the notion of derivation. Intuitively a derivation (or proof) is a (finite) sequence of tagged literals $P=(P(1), \ldots, P(i))$ (or, $P(1 . . n)$ ), where every element (a conclusion) is either one of the facts, or it has been obtained by previous steps by applying some rules. A conclusion of a defeasible theory $D$ is a tagged literal and can have one of the following forms: 
1. $+\Delta q$, which means that $q$ is definitely provable in $D$, i.e., there is a definite proof for $q$ (a proof using facts, and strict rules only);

2. $-\Delta q$, which means that $q$ is definitely not provable in $D$ (i.e., a definite proof for $q$ does not exist);

3. $+\partial q$, which means that $q$ is defeasibly provable in $D$;

4. $-\partial q$, which means that $q$ is not defeasibly provable, or refuted in $D$.

The definition of $\Delta$ describes just forward chaining of strict rules. Literal $q$ is definitely provable if either is a fact, or there is a strict rule for $q$, whose antecedents have all been definitely proved.

$+\Delta$ : If $P(n+1)=+\Delta q$ then

(1) $q \in F$ or

(2) $\exists r \in R_{S}[q] \forall a \in A(r):+\Delta a \in P(1 . . n)$.

The negative proof conditions for $\Delta$ (the same thing will hold for $\partial$ ) are the strong negation of the positive counterpart: this is closely related to the function that simplifies a formula by moving all negations to an innermost position in the resulting formula, and replaces the positive tags with the respective negative tags and the other way around $[17,18]$.

$-\Delta:$ If $P(n+1)=-\Delta q$ then

(1) $q \notin F$ and

(2) $\forall r \in R_{S}[q] \exists a \in A(r):-\Delta a \in P(1 . . n)$.

The proof conditions just given are meant to represent forward chaining of facts and strict rules $(+\Delta)$, and that it is not possible to obtain a conclusion just by using forward chaining of facts and strict rules $(-\Delta)$.

The proof conditions for $\pm \partial$ are as follows:

$+\partial$ : If $P(n+1)=+\partial q$ then either

(1) $+\Delta q \in P(1 . . n)$, or

(2) $(2.1)-\Delta \sim q \in P(1 . . n)$ and

(2.2) $\exists r \in R_{s d}[q]$ such that $\forall a \in A(r):+\partial a \in P(1 . . n)$ and

(2.3) $\forall s \in R[\sim q]$ either

(2.3.1) $\exists a \in A(s)$ such that $-\partial a \in P(1 . . n)$, or

(2.3.2) $\exists t \in R_{s d}[q]$ such that

$\forall a \in A(t):+\partial a \in P(1 . . n)$ and $t>s$.

$-\partial$ : If $P(n+1)=-\partial q$ then

(1) $+\Delta q \notin P(1 . . n)$ and either

(2) (2.1) $+\Delta \sim q \in P(1 . . n)$, or

(2.2) $\forall r \in R_{s d}[q] \exists a \in A(r):-\partial a \in P(1 . . n)$ or

(2.3) $\exists s \in R[\sim q]$ such that

(2.3.1) $\forall a \in A(s):+\partial a \in P(1 . . n)$ and

(2.3.2) $\forall t \in R_{s d}[q]$ either

$\exists a \in A(t):-\partial a \in P(1 . . n)$, or $t \ngtr s$. 
Literal $q$ is defeasibly provable if $q$ is already definitely provable, or we argue by using the defeasible part of the theory. In this last case, there must exist an applicable strict or defeasible rule for $q$, while every attack is either discarded, or defeated by a stronger rule through $>$. A rule is merely applicable whenever each literal in the set of antecedents has already been proved (according to the appropriate conditions), while a rule is discarded when at least one of the premises has been previously disproved. Conditions for $-\partial$ show that any systematic attempt to defeasibly prove the conclusion fails.

In Defeasible Logic, the defeasible provability depends on the notion of team defeater: rules for a conclusion 'fight as a team' against rules for the opposite conclusion. The winning mechanism is given by the superiority relation. Suppose rules $r_{1}$ and $r_{2}$ support $q$, rules $s_{1}$ and $s_{2}$ support $\sim q,>=\left(r_{1}, s_{1}\right),\left(r_{2}, s_{2}\right)$. We conclude $+\partial q$ and $-\partial \sim q$, since both rules for $\sim q$ are defeated. If we now change the superiority relation to $>=\left(r_{1}, s_{1}\right),\left(r_{2}, s_{1}\right)$, we conclude $-\partial q$ and $-\partial \sim q$, since now neither $r_{1}$, nor $r_{2}$ is stronger than $s_{2}$ and we cannot solve the conflict. Note that the superiority relation is not confined to defeasible rules only: this is so because we can use strict rules to derive defeasible conclusions (condition (2.2) of $+\partial$ ) when at least one of the antecedents has been proved as defeasible. In that scenario, the superiority relation may play a role. This is not the case for definite provability: if two strict rules for opposite conclusions are applicable, they will both draw a conclusion, giving rise to an inconsistency. This is discussed later in Proposition 1, which defines when a defeasible theory is inconsistent.

The conditions for $\pm \Delta$ and $\pm \partial$ are related by the Principle of Strong Negation introduced in [17]. The key idea behind this principle is that conclusions labelled with a negative proof tag are the outcome of a constructive proof that the corresponding positive conclusion cannot be obtained (and the other way around). We shall illustrate the mechanism of proofs/derivations and proof conditions with the following example.

Example 2. Consider $D=\left(\{a, b\}, R,\left\{\left(r_{4}, r_{5}\right)\right\}\right)$, with $R=\{$

$$
\begin{array}{ll}
r_{1}: a \rightarrow c & r_{2}: \Rightarrow d \\
r_{3}: \Rightarrow e & r_{4}: c, d \Rightarrow p \\
\left.r_{5}: b, e \Rightarrow \neg p\right\} . &
\end{array}
$$

A proof for $+\partial p$ is the sequence $(+\Delta a,+\partial a,+\Delta c,+\partial c,-\Delta \neg d,+\partial d,-\Delta \neg p,+\partial p)$, while a proof for $-\partial \neg p$ is the sequence $(+\Delta a,+\partial a,+\Delta c,+\partial c,+\Delta b,+\partial b,-\Delta \neg e,+\partial e,-\Delta \neg d,+\partial d$, $-\Delta \neg p,+\partial p,-\partial \neg p)$. Here, rule $r_{4}$ and $r_{5}$ lead to opposite conclusions. The stall is resolved by the superiority relation, that allows us to prove $+\partial p$. If no superiority relation had been given, we would have both $-\partial p$ and $-\partial \neg p$.

As usual, given a proof tag \#, a literal $p$, and a theory $D$, we use $D \vdash \pm \# p$ to denote that there is a proof $P$ in $D$ where for some line $i, P(i)= \pm \# p$. Alternatively, we say that $\pm \# p$ holds in $D$, or simply $\pm \# p$ holds when the theory is clear from the context.

The set of positive and negative conclusions is called an extension. Formally,

Definition 1 Given a defeasible theory $D$, the defeasible extension of $D$ is defined as

$$
E(D)=(+\Delta,-\Delta,+\partial,-\partial),
$$

where, for $\# \in \Delta, \partial, \pm \#=l: l$ appears in $D$ and $D \vdash \pm \# l$. 
Note that condition (2.2) of $+\partial$ states that a strict rule can be used to draw a defeasible conclusion. This implies that two strict rules may be in a superiority relation with each other, but this will have a role only when considering $\pm \partial$.

In this paper, we shall not make use of strict rules (since every revision changes only the priority among defeasible rules and defeaters), nor defeaters. The restriction does not result in any loss of generality: (1) the superiority relation does not play any role in proving definite conclusions, and (2) for defeasible conclusions Antoniou et al. [16] prove that it is always possible to remove (a) strict rules from the superiority relation and (b) defeaters from the theory to obtain an equivalent theory without defeaters and where the strict rules are not involved in the superiority relation.

Accordingly, and due to the nature of the revision operators discussed in this paper, we shall consider extensions restricted to the defeasible part only. In fact, it is not possible to change a defeasible theory to modify its strict conclusions by revising the superiority relation: the only way to modify them is to operate on the set of strict rules (i.e., addition or removal), or on their applicability by modifying the set of facts.

Therefore, in the following, we restrict $E(D)$ to $(+\partial,-\partial)$. A common notation in Defeasible Logic is: $E^{+}(D)=+\partial$ and $E^{-}(D)=-\partial$. We thus have $D \vdash+\partial p$ iff $p \in E^{+}(D)$. Referring to Example $2, E^{+}(D)=\{a, b, c, d, e, p\}$, while $E^{-}(D)=\{\neg p\}$.

We now address the issue of defining when a defeasible theory is (in)consistent.

Definition 2 A defeasible theory $D$ is inconsistent iff there exists a literal $p$ such that $D \vdash+\partial p$ and $D \vdash+\partial \neg p$.

The inference mechanism of Defeasible Logic does not allow us to derive inconsistencies unless the monotonic (strict) part of the logic is inconsistent, or when the superiority relation contains a cycle (or in other words when we can say that a rule is at the same time stronger and weaker than another rule), as stated below.

Proposition 1 [16, Proposition 3.3] Let D be a theory where the transitive closure of the superiority relation is acyclic. If $D$ proves $D \vdash+\partial p$ and $D \vdash+\partial \neg p$, then $D \vdash+\Delta p$ and $D \vdash+\Delta \neg p$.

Defeasible Logic is para-consistent in the sense that a contradiction does not trivialise the set of conclusions that can be derived from a theory. Consider the following example.

Example 3. Consider $D=\left(\emptyset, R,\left\{\left(r_{1}, r_{2}\right),\left(r_{2}, r_{1}\right),\left(r_{4}, r_{3}\right)\right\}\right)$, with $R=\{$

$$
\begin{array}{ll}
r_{1}: \Rightarrow p & r_{2}: \Rightarrow \neg p \\
r_{3}: p \Rightarrow q & \left.r_{4}: \Rightarrow \neg q\right\} .
\end{array}
$$

Given the cycle in the superiority relation we have both $D \vdash+\partial p$ and $D \vdash+\partial \neg p$. However, we have $D \vdash+\partial \neg q$ and $D \vdash-\partial q$.

The next example is intended only to introduce the graphical notation adopted in the rest of the paper. 
Example 4. Consider $D=\left(\emptyset, R,\left\{\left(r_{1}, r_{4}\right),\left(r_{5}, r_{3}\right)\right\}\right)$, with $R=\{$

$$
\begin{array}{ll}
r_{1}: \Rightarrow a & r_{7}: \Rightarrow b \\
r_{2}: a \Rightarrow c & r_{8}: \Rightarrow \neg c \\
r_{3}: c \Rightarrow d & r_{9}: \Rightarrow \neg b \\
r_{4}: \Rightarrow \neg a & r_{10}: \Rightarrow e \\
r_{5}: \Rightarrow \neg d & r_{11}: \Rightarrow f \\
r_{6}: \neg d \Rightarrow p & \left.r_{12}: a, g \Rightarrow \neg f\right\} .
\end{array}
$$

To improve readability, hereafter we use the following graphical notation:

$$
\begin{aligned}
& \Rightarrow_{r_{1}} \quad a \Rightarrow_{r_{2}} \quad c \Rightarrow_{r_{3}} d \\
& \vee \\
& \Rightarrow_{r_{4}} \neg a \quad{ }_{r_{5}} \neg d \Rightarrow_{r_{6}} p \\
& \Rightarrow_{r_{7}} \quad b \Rightarrow_{r_{8}} \neg c \\
& \Rightarrow_{r_{9}} \neg b \\
& \Rightarrow_{r_{10}} e \Rightarrow_{r_{11}} f \\
& a, g \Rightarrow_{r_{12}} \neg f,
\end{aligned}
$$

where the $\wedge$ and $\vee$ symbols in the graphical representation of a theory represent tuples of the superiority relation $>$ (thus, $\vee$ in the second line in between $r_{1}$ and $r_{4}$ stands for $\left(r_{1}, r_{4}\right) \in>$, while $\wedge$ between $r_{3}$ and $r_{5}$ stands for $\left.\left(r_{5}, r_{3}\right) \in>\right)$; the labels of the rules are denoted by the subscript $r_{i}$ to the symbol $\Rightarrow$.

\subsection{New proof tags}

We now present the two additional proof tags $\pm \Sigma$ and $\pm \varphi$ : they will be helpful to simplify the technical presentation and results discussed in the next sections. $+\Sigma q$ means that there exists a reasoning chain supporting $q$, while $+\varphi q$ means that there exists a proof (i.e., a reasoning chain) made of rules such that, for every element in it (say $a$ ), there is no supporting chain for the opposite conclusion ( $-\Sigma a$ holds). Proof tag $+\varphi q$ will also play a fundamental role in defining the concept of tautology later on, and ultimately will help us in proving the NP-completeness results.

enumi4

1. $\quad-r_{1}, r_{2}, r_{3}$ form a chain supporting literal $d(+\Sigma d)$.

2. $-\Sigma q$, which means there is no reasoning chain supporting $q$.

- Since there are no rules for literal $\neg p$, then we have $-\Sigma \neg p$.

3. $+\sigma q$, which means there exists a reasoning chain supporting $q$ made of rules never attacked by any applicable rule for the opposite conclusion.

- $r_{1}, r_{2}$ and $r_{7}, r_{8}$ are two undefeated chains for $c$ and $\neg c$, respectively; Thus, we have $+\sigma c,+\sigma \neg c$.

4. $-\sigma q$, which means that, in every reasoning chain supporting $q$, at least one rule is attacked by an applicable rule for the opposite conclusion.

- Every chain for $d$ is defeated ( $-\sigma d$, notice that there exists only one in this case).

5. $+\omega q$, which means there exists an applicable rule supporting $q$. 
- In the chain $r_{1}, r_{2}, r_{3}$, only rule $r_{3}$ is defeated, hence $+\omega d$ holds.

6. $-\omega q$, which means that in every rule supporting $q$, at least one of its antecedents is defeasibly refuted.

- Since $+\partial b$ does not hold, we can conclude $-\omega \neg c$.

7.

$-\varphi q$, which means that there exists a rule $r$ with $C(r)=a$ in every reasoning chain supporting $q$ such that there exists $s$ with $C(s)=\neg a$. ( $s$ implies that a point of possible revision exists.)

- $r_{4}$ supports $\neg a$, hence we have $-\varphi a$.

These proof tags are formally defined by the following proof conditions ${ }^{3}$.

$+\Sigma$ : If $P(n+1)=+\Sigma q$ then

(1) $+\Delta q \in P(1 . . n)$ or

(2) $\exists r \in R_{s d}[q]$ such that $\forall a \in A(r):+\Sigma a \in P(1 . . n)$

$-\Sigma$ : If $P(n+1)=-\Sigma q$ then

(1) $+\Delta q \notin P(1 . . n)$ and

(2) $\forall r \in R_{s d}[q]: \exists a \in A(r)$ such that $-\Sigma a \in P(1 . . n)$

Definitions of $\Sigma$ formalise the concept of supporting chain for a given literal $q$. For instance, if $-\Sigma p$ holds for a literal $p$, then no modification on the superiority relation are possible to prove $p$. (We could then only intervene on the facts or the rules; see [19] for theory revision operations on legal reasoning.)

$+\sigma:$ If $P(n+1)=+\sigma q$ then

(1) $+\Delta q \in P(1 . . n)$ or

(2) $\exists r \in R_{s d}[q]$ such that

(2.1) $\forall a \in A(r):+\sigma a \in P(1 . . n)$ and

(2.2) $\forall s \in R[\sim q] \exists a \in A(s)$ such that

$-\partial a \in P(1 . . n)$ or $s \ngtr r$.

$-\sigma$ : If $P(n+1)=-\sigma q$ then

(1) $+\Delta q \notin P(1 . . n)$ and

(2) $\forall r \in R_{s d}[q]$ :

(2.1) $\exists a \in A(r)$ such that $-\sigma a \in P(1 . . n)$ or

(2.2) $\exists s \in R[\sim q]$ such that

$\forall a \in A(s):+\partial a \in P(1 . . n)$ and $s>r$.

Note that the definitions given above for $\pm \sigma$ are weak forms of the notion of support proposed in [20,17] for the definition of an ambiguity propagating variant of Defeasible Logic, in the sense that these definitions are less selective than the ones of [20].

As speaking of possible superiority revisions, the chains that allow to state $+\sigma p$ may be good candidates to defeasibly prove $p$ as they represent undefeated elements.

\footnotetext{
${ }^{3}$ Again, the negative counterparts are obtained by the principle of strong negation. An important consequence of using this principle to formulate the conditions for asserting tagged literals is that for any literal $p$ and any proof tag \#, it is not possible to have both $+\# p$ and $-\# p$. The interested reader is referred to $[17,18]$.
} 
$+\omega$ : If $P(n+1)=+\omega q$ then

(1) $+\Delta q \in P(1 . . n)$ or

(2) $\exists r \in R_{s d}[q]$ such that $\forall a \in A(r):+\partial a \in P(1 . . n)$.

$-\omega$ : If $P(n+1)=-\omega q$ then

(1) $+\Delta q \notin P(1 . . n)$ and

(2) $\forall r \in R_{s d}[q]: \exists a \in A(r)$ such that $-\partial a \in P(1 . . n)$.

Proof tag $+\omega p$ represents a defeasible proof for $p$ that only fails on the last derivation step. Possible modifications can thus focus on this last step instead of considering the whole chain. (Considering that in Defeasible Logic, a team defeater of rules competes against another to prove/refute a given literal, focusing on the last step may involve considering all, or a part of such rules.)

$+\varphi$ : If $P(n+1)=+\varphi q$ then

(1) $+\Delta q \in P(1 . . n)$ or

(2) $\exists r \in R_{s d}[q]$ such that

(2.1) $\forall a \in A(r):+\varphi a \in P(1 . . n)$ and

(2.2) $\forall s \in R[\sim q]: \exists a \in A(s)$ such that $-\Sigma a \in P(1 . . n)$.

$-\varphi$ : If $P(n+1)=-\varphi q$ then

(1) $+\Delta q \notin P(1 . . n)$ and

(2) $\forall r \in R_{s d}[q]$ :

(2.1) $\exists a \in A(r)$ such that $-\varphi a \in P(1 . . n)$ or

(2.2) $\exists s \in R[\sim q]$ such that $\forall a \in A(s):+\Sigma a \in P(1 . . n)$.

The definition of $+\varphi$ ensures that it is not possible to have a counter-argument against any literal within such a chain. No modifications on the superiority relation are therefore possible to reject a literal tagged with $+\varphi$.

Consider Example 4: the sequence of rules ' $r_{1} r_{2} r_{3}$ ' forms a chain supporting literal $d$, hence $D \vdash+\Sigma d$; there are no rules for $g$, this makes $r_{12}$ to always be discarded, thus $D \vdash-\Sigma g$ as well as $D \vdash-\Sigma \neg f$. Additionally, there are no rules for $\neg e$, and the only rule for $\neg f, r_{12}$, is such that for its only antecedent $(g)$, we have $D \vdash-\Sigma g$; thus $d \vdash+\varphi e$, $D \vdash+\varphi f$, and $D \vdash-\varphi \neg f$ since $D \vdash-\varphi g$.

Given the above definitions, we can derive the series of implications reported below in Figure 1(a)-(b) using techniques presented in [21].

One could be tempted to say that $+\sigma$ implies $+\omega$ (and symmetrically, that $-\omega$ implies $-\sigma)$. That is not the case. Indeed, if we consider theory $D$ of Example 4, we have: (i.) $+\omega d$ and $-\sigma d$, (ii.) $+\sigma \neg c$ and $-\omega \neg c$.

To better explain how the new proof tags behave, we report in Table 1 the set of all conclusions. For each literal, we only report the proof tag which is minimal with respect to the orderings given in Figure 1. For example, $+\partial a$ means that we prove $+\omega a,+\sigma a,+\Sigma a$, but $-\varphi a$. If no tag is reported, then it is not possible to derive the literal with any tags with respect to the ordering given in Figure 1. 


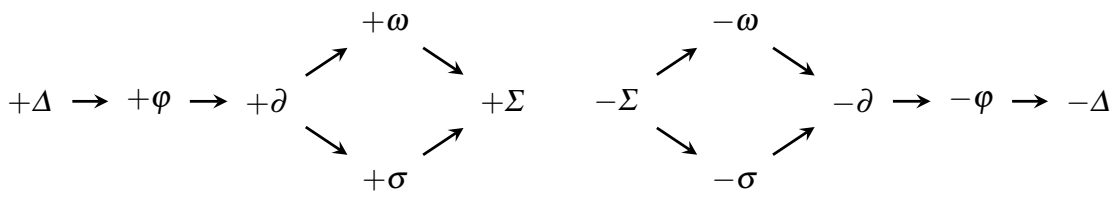

(a) Positive implication chain

(b) Negative implication chain

Fig. 1: Implication chains.

\begin{tabular}{|c|c|c|c|c|c|c|}
\hline$a$ & $b$ & $c$ & $d$ & $e$ & $f$ & $p$ \\
\hline$++\partial$ & $+\sigma$ & $+\partial$ & $+\omega$ & $+\varphi$ & $+\varphi$ & $+\partial$ \\
\hline$--\varphi$ & $-\partial$ & $-\partial$ & $-\sigma$ & & & $-\varphi$ \\
\hline$\neg a$ & $\neg b$ & $\neg c$ & $\neg d$ & $\neg e$ & $\neg f$ & $\neg p$ \\
\hline$++\omega$ & $+\sigma$ & $+\sigma$ & $+\partial$ & & & \\
\hline$--\partial$ & $-\partial$ & $-\omega$ & $-\varphi$ & -2 & -2 & -2 \\
\hline
\end{tabular}

Table 1: Conclusions for literals in Example 4.

\subsection{Properties}

We now present some first theoretical results which will be useful throughout the rest of the paper.

Next Proposition 2 formally justifies such a claim, and, specifically, states that for every literal $q$ such that $D \vdash+\varphi q$, no applicable rules supporting $\sim q$ exist. The result is not guaranteed if $+\Delta q$ holds.

Proposition 2 Given a consistent defeasible theory $D$ and a literal $p$, if $D \vdash+\varphi p$ and $D \vdash-\Delta p$, then $D \vdash-\Sigma \sim p$.

Proof. The proof straightly follows from condition (2.2) of $+\varphi$. This condition must hold for each element in the chain, as well as for $p$.

The next proposition formally states that, given a defeasibly proved literal $p$ and a chain leading to $\sim p$ with all the antecedents defeasibly proved, such a chain is defeated by a priority rule on the last proof step. (Equivalently, all rules proving $\sim p$ are defeated; the attacks come from the team defeater supporting $p$.)

Proposition 3 Given a consistent defeasible theory $D$, if $D \vdash+\partial p$ and $D \vdash+\omega \sim p$ for a literal $p$ (with $p \notin F$ ), then $D \vdash-\sigma \sim p$.

Proof. By definition of $+\partial$, we have that the condition below

(2.3) $\forall s \in R[\sim p]$ either 


$$
\begin{aligned}
& \text { (2.3.1) } \exists a \in A(s):-\partial a \in P(1 . . n) \text { or } \\
& \text { (2.3.2) } \exists t \in R_{s d}[p] \text { such that } \\
& \forall a \in A(t):+\partial a \in P(1 . . n) \text { and } t>s
\end{aligned}
$$

holds for $p$. In fact condition (2.3.2) has to be true since we know condition (2.3.1) is not, because

$$
\left.\begin{array}{c}
D \vdash+\partial p \text { implies } \exists r \in R[p] . \forall a \in A(r):+\partial a \\
D \vdash+\omega \sim p \text { implies } \exists s \in R[\sim p] . \forall a \in A(s):+\partial a
\end{array}\right\} \text { thus }
$$

This is condition (2.2) for $-\sigma \sim p$. Moreover, since all the premises of $\sim p$ are defeasibly proved by hypothesis and we proved that the chain is defeated, condition (2.3.2) of $+\partial$ states that this is the case only if there exists an applicable rule supporting $p$ stronger than the rule for $\sim p$.

We now capture the concept of a derivation based on a contradiction. To do so, we firstly need to introduce the notion of dependency; secondly, we look at the notion of $\partial$-unreachability, which defines literals with a derivation based upon an inconsistency.

Definition 3 Let $a$ and $b$ be two literals. Then $a$ depends on $b$ iff (1) $b=a$, or (2) $\forall r \in R[a]$ either (2.1) $b \in A(r)$, or (2.2) $\exists c$ such that $c \in A(r)$ and $c$ depends on $b$.

We say that a depends on $b$ with degree 1 if a depends on $b$, and there exists a rule $r$, such that $C(r)=a$ and $b \in A(r)$; $a$ depends on $b$ with degree $n+1$ if $a$ depends on $b$, and there exists a literal $c$ such that a depends on $c$ with degree 1 and $c$ depends on $b$ with degree $n$.

Roughly, if literal $a$ depends on literal $b$, then $b$ is present in every chain leading to $a$. Referring to Example 4, $d$ depends on $a$ with degree 2 .

The following result shows that a defeasibly proved literal also implies the provability of all literals it depends on. In other words, the property of dependency propagates backwards the defeasible provability of literals.

Proposition 4 Given a defeasible theory $D$ such that $D \vdash+\partial p$ and $p$ depends on $q$, then $D \vdash+\partial q$.

Proof. The proof is by case inspection of Definition 15. If clause (1) holds, the claim trivially follows. For the other cases, the proof is by induction on the dependency degree.

Base case: $p$ depends on $q$ with degree 1 implies that, for every rule $r \in R[p], q \in$ $A(r)$ is the case. Thus, $+\partial p$ implies that (at least) one of such rules is applicable, and we conclude $D \vdash+\partial q$.

Inductive step: suppose that the property holds up to degree $n$, and that $p$ depends on $q$ with degree $n+1$. By Definition 15, there exists a literal $c$ such that $p$ depends on $c$ with degree 1 . Hence, $D \vdash+\partial c$ (given $D \vdash+\partial p$ by hypothesis) and $c$ depends on $q$ with degree $n$. By the inductive hypothesis, we now conclude $D \vdash+\partial q$. 
The next definition identifies literals that depend on contradictions. For instance, consider the theory with the one rule:

$$
a, \neg a \Rightarrow p .
$$

To prove $+\partial p$ we need both $+\partial a$ and $+\partial \neg a$, but this is the case iff the theory is inconsistent. We also have to cater for situations where the dependency is not direct, like

$$
a \Rightarrow b \quad \neg a, b \Rightarrow p .
$$

Definition 4 A literal $p$ is $\partial$-unreachable iff $\forall r \in R[p]$ either (1) $\exists l, \exists a, b \in A(r)$ such that (1.1) a depends on $l$ and (1.2) $b$ depends on $\sim l$, or (2) $\exists d \in A(r)$ such that $d$ is $\partial$-unreachable. Otherwise, we define $p$ to be $\partial$-reachable.

The next result formalises the relationship between $\partial$-unreachable literals and inconsistent theories.

Proposition 5 Given a theory $D$, let $p$ be a $\partial$-unreachable literal. If $D \vdash+\partial p$, then $D$ is inconsistent.

Proof. The proof is by induction on the number of $\partial$-unreachable literals in a derivation.

Base case: $p$ is the only $\partial$-unreachable literal in its derivation. By $D \vdash+\partial p$, there exists (at least) one applicable rule for $p$. By Definition 4 , in every rule for $p$ there are two antecedents, viz. $a$ and $b$, such that $a$ depends on a given literal $l$ while $b$ depends on $\sim l$. Thus, we have both $+\partial a$ and $+\partial b$. By Proposition 8, we have $D \vdash+\partial l$ and $D \vdash+\partial \sim l$, and we conclude that $D$ is inconsistent.

Inductive step: we assume that the property holds up to $n \partial$-unreachable literals, and $p$ is the $(n+1)^{t h} \partial$-unreachable literal. Beside the case we examined in the inductive base, we now need to consider that the antecedent of a rule contains a $\partial$-unreachable literal $d$, and that $D \vdash+\partial d$. Thus, $d$ falls under the inductive hypothesis, and we conclude that $D$ is inconsistent.

Proof tag $\varphi$ and condition of $\partial$-reachability are not useful per se but must be seen in the bigger picture of understanding how hard is the problem of deciding whether a given literal is a tautology within the framework of changing the superiority relation only. The relative discussion is therefore postponed until after we prove the NP-completeness result and in Section 7 during the AGM analysis.

We end this section by formalising in our logic a real life example, based on a case disputed in an Italian court first and then appealed to the European Court House in Strasburg, 2012. The example illustrates a general property of legal reasoning: the resolution of a dispute can be represented as the change of the superiority relation of the defeasible theory encoding the (relevant) legal rules.

Example 5. A couple can have offspring but, since both the mother and father are affected of cystic fibrosis, they know that there is a extremely high probability that every child of them will be affected by the same genetic anomaly. Since they want their offspring to be healthy, they request for medically assisted reproduction techniques. Their 
case is disputed first in an Italian Court where the judge has to establish which between Art. 4 of Italian Legislative Act 40/2004 ( $r_{0}$ and $r_{1}$ ) and the 'Standard Common Medical Practice' $\left(r_{3}\right)$ in force in 15 countries of the EU prevails over the other.

The couple is indeed able to produce embryos and they cannot be considered as sterile $\left(r_{2}\right)$. This makes both Art. 4 and the Standard Common Medical Practice to be applicable to their case. The judge argues in favour of $r_{1}$ based on lex superior principle and refuses their request: this principle applies since Art. 4 Act 40/2004 is a legal rule while $r_{3}$ has a judicial validity but it is not a proper legal rule.

$$
\begin{aligned}
& F= \text { Embryo, GeneticAnomalies } \\
& R=\left\{r_{0}: \neg \text { CandidateInVitroFertilization } \Rightarrow \neg\right. \text { Techniques, } \\
& r_{1}: \neg \text { Sterility } \Rightarrow \neg \text { CandidateInVitroFertilization, } \\
& r_{2}: \text { Embryo } \Rightarrow \neg \text { Sterility, } \\
& \quad r_{3}: \neg \text { Sterility, GeneticAnomalies } \Rightarrow \text { CandidateInVitroFertilization, } \\
& r_{4}: \neg \text { Sterility, GeneticAnomalies } \Rightarrow \neg \text { Healthy } \\
&\left.r_{5}: \text { GeneticAnomalies, CandidateInVitroFertilization } \Rightarrow \text { Healthy }\right\} \\
&>=\left\{\left(r_{1}, r_{3}\right)\right\} .
\end{aligned}
$$

DBLP : conf/icail/Governatori

The couple appeals to the European Court for Human Rights. The European Court establishes that not permitting the medical techniques would demote the goal of family health promoted by Article 8 of the Convention and, therefore, states that the couple is actually allowed to have the medically assisted reproduction procedure. In our example, $r_{3}$ promotes the goal of family health $\left(r_{5}\right)$, and thus the European Court inverts the priority between $r_{1}$ and $r_{3}$ based both on lex superior and lex specialis $\left(>^{\prime}=\left\{\left(r_{3}, r_{1}\right)\right\}\right)$. In here, the lex superior principle applies because $r_{3}$ is an European directive, while the lex specialis principle applies because $r_{3}$ covers a more specific case than $r_{1}$.

\section{Changing Defeasible Preferences}

We shall now analyse the processes of revision in a defeasible theory, when no changes to rules and facts are allowed. Henceforth, when no confusion arises, every time we speak about a (revision) transformation, we shall assume a (revision) transformation acting on the superiority relation only.

A good starting point for our investigation is to focus on the corresponding decision problem (i.e., answering the question: 'Is it possible to modify the state of a literal in a defeasible theory by only changing the superiority relation?') and characterise it in a formal way. We name such a problem the Defeasible Superiority Changing Problem, shortly DSC Problem. More formally,

Definition 5 (DSC Problem) Given a defeasible theory $D=(F, R,>)$ and a literal $p$ such that $D \vdash+\partial p(D \vdash-\partial p)$, is it possible to obtain $D^{\prime}=\left(F, R,>^{\prime}\right)$ such that $D^{\prime} \vdash$ $-\partial p\left(\right.$ resp. $\left.D^{\prime} \vdash+\partial p\right)$ ?

\footnotetext{
${ }^{4}$ Art. 4 of Italian Legislative Act 40/2004: "The recourse to medically assisted reproduction techniques is allowed only [...] in the cases of sterility".
} 
This is the problem shown in the example at the end of the previous section. In the rest of the section, we show that the decision problem at hand is computationally hard.

\subsection{NP-Completeness}

We start by introducing some additional terminology. Definition 6 below constructs a defeasible theory starting from a fixed set of rules and an empty set of facts. This formulation limits the revision problem to preference changes, notwithstanding the particular instance of the superiority relation.

Definition 6 Given a set of defeasible rules $R$, a defeasible theory $D$ is based on $R$ iff

$$
D=(\emptyset, R,>)
$$

for some superiority relation $>$.

Next Definition 7 specifies the possible relationships between a literal and all the theories based on set of rules $R$.

Definition 7 Given a set of defeasible rules $R$, a literal $p$ is

1. $>-R$-tautological iff for all theories $D$ based on $R, D \vdash+\partial p$.

2. $>-R$-non-tautological iff there exists a theory $D$ based on $R$ such that $D \nvdash+\partial p$.

3. $>-R$-refutable iff there exists a theory $D$ based on $R$ such that $D \vdash-\partial p$.

4. $>-R$-irrefutable iff for all theories $D$ based on $R, D \nvdash-\partial p$.

The notion of $>-R$-irrefutable represents the negative counterpart of $>-R$-tautological; we have the same symmetry between $>-R$-refutable and $>-R$-non-tautological.

If $p$ is $>-R$-tautological, then no assignment of the superiority relation allows to disprove $p$, and we cannot revise it. On the contrary, if there exists an instance of the superiority relation such that $-\partial p$ holds, then $p$ is $>-R$-refutable.

Example 6. Consider $D=(\emptyset, R, \emptyset)$, with $R=\left\{r_{0}: \emptyset \Rightarrow a, r_{1}: b \Rightarrow b, r_{3}: \emptyset \Rightarrow c, r_{4}: \emptyset \Rightarrow\right.$ $\neg c$ \} Here, $a$ is $>-R$-tautological (and thus $>-R$-irrefutable), $\neg a$ is $>-R$-refutable, $b$ is $>-R$-irrefutable but not $>-R$-tautological, both $c$ and $\neg c$ are both $>-R$-non-tautological

To prove that the DSC Problem is NP-complete, we reduce the 3-SAT Problem to ours. Specifically, we map a 3-SAT formula to a set of rules $R$ and check whether a specific literal appearing $R$ is $>-R$-tautological. Definition 8 exhibits the reduction adopted.

Definition 8 Given a 3-SAT formula $\Gamma=\bigwedge_{i=1}^{n} C_{i}$ such that $C_{i}=\bigvee_{j=1}^{3} a_{j}^{i}$, we define the $\Gamma$ transformation as the operation that maps $\Gamma$ into the following set of defeasible rules

$$
\begin{aligned}
R_{\Gamma}=\left\{r_{i j}^{a}: \Rightarrow a_{j}^{i}\right. \\
r_{i j}: a_{j}^{i} \Rightarrow c_{i} \\
r_{\sim i}: \Rightarrow \sim c_{i} \\
\left.r_{i}: \sim c_{i} \Rightarrow p\right\} .
\end{aligned}
$$


It is immediate to verify that the mapping proposed in the definition above is polynomial in the number of literals appearing in the 3-SAT formula $\Gamma$, since every literal in the input has associated exactly four rules in output.

The second step of the proof construction is to ensure the correctness of the proposed mapping. Proposition 6 and Lemma 10 guarantee that any theory obtained through a $\Gamma$-transformation provides an answer. These results are also intended to establish relationships between the notions of tautological and refutable given in Definition 7.

Definition 9 A defeasible theory $D$ is decisive iff for every literal $p$ in $D$ either $D \vdash$ $+\partial p$, or $D \vdash-\partial p$.

Proposition 6 Given a defeasible theory $D$, if the atom dependency graph ${ }^{5}$ of $D$ is acyclic, then $D$ is decisive.

Proof. For a detailed definition of atom dependency graph and a complete proof of the claim, the interested reader should refer to [22].

Lemma 10 Any defeasible theory $D$ based on $R_{\Gamma}$ of Definition 8 (for any $\Gamma$ ) is decisive.

Proof. It is easy to verify by case inspection that the atom dependency graph is acyclic.

We are now ready to introduce the main result of NP-completeness. First things first, we have to prove that the DSC Problem is in NP. Second, we show that it is NP-hard by exploiting the formulation of the 3-SAT problem and the transformation proposed in Definition 8.

Theorem 11 The problem of determining the revision of a defeasible literal by changing the preference relation is $\mathrm{NP}$-complete.

Proof. The proof that the DSC Problem is in NP is straightforward. Given a defeasible theory $D=(F, R,>)$ and a literal $p$ to be revised, an oracle guesses a revision (in terms of a new preference relation $>^{\prime}$ applied to $D$ ) and checks whether the state of $p$ has changed in $E\left(D^{\prime}\right)\left(D^{\prime}=\left(F, R,>^{\prime}\right)\right)$ with respect to $E(D)$. The complexity of this check is bound to the computation of $E(D)$ and $E\left(D^{\prime}\right)$, which [23] proves to be linear in the order of the theory.

To prove the NP-hardness, given a 3-SAT formula $\Gamma=\bigwedge_{i=1}^{n} C_{i}$ such that $C_{i}=\bigvee_{j=1}^{3} a_{j}^{i}$, a defeasible theory $D$ based on the set of defeasible rules $R_{\Gamma}$ obtained by $\Gamma$-transformation, and a literal $p$ in $D$, we show that:

(1) if $p$ is $>-R_{\Gamma}$-tautological, then $\Gamma$ is not satisfiable;

(2) if $p$ is $>-R_{\Gamma}$-non-tautological, then $\Gamma$ is satisfiable.

\footnotetext{
${ }^{5}$ In the Atom Dependency Graph the atomic propositions are the nodes, and there is a directed edge between nodes if there is a rule containing the source or its negation in the body, and the target or its negation in the head.
} 
(1) Lemma 10 allows us to reformulate the contrapositive using $>-R_{\Gamma}$-refutable. If $\Gamma$ is satisfiable, then there exists an interpretation $I$ such that

$$
\begin{aligned}
I \vDash \Gamma & \Longleftrightarrow I \vDash \bigwedge_{i=1}^{n} C_{i} \\
& \Longleftrightarrow I \vDash C_{1} \text { and } \ldots \text { and } I \vDash C_{n} \\
& \Longleftrightarrow I \vDash \bigvee_{j=1}^{3} a_{j}^{1} \text { and } \ldots \text { and } I \vDash \bigvee_{j=1}^{3} a_{j}^{n}
\end{aligned}
$$

Thus, for each $i$, there exists $j$ such that $I \vDash a_{j}^{i}$.

We build a defeasible theory $D^{\prime}=\left(\emptyset, R_{\Gamma},>^{\prime}\right)$ as follows. If there exists a literal $b_{k}^{l}$ such that $b_{k}^{l}=\sim a_{j}^{i}$, then $\left(r_{i j}^{a}, r_{l k}^{b}\right)$ is in $>^{\prime}$. It follows that, by construction, $D^{\prime} \vdash+\partial a_{j}^{i}$. This means that every rule $r_{i j}$ is applicable and it is not weaker than the corresponding rule $r_{\sim i}$. Hence, we have $-\partial \sim c_{i}$, for all $i$. Consequently, each rule $r_{i}$ for $p$ is discarded and we conclude $D^{\prime} \vdash-\partial p$. Accordingly, $p$ is $>-R_{\Gamma}$-refutable.

(2) Again, due to Lemma 10, every theory based on $R_{\Gamma}$ is decisive. Thus, $p$ is $>-R_{\Gamma^{-}}$ refutable. Accordingly, there exists a theory $D^{\prime}=\left(\emptyset, R_{\Gamma},>^{\prime}\right)$ such that $D^{\prime} \vdash-\partial p$. Given that $R_{\Gamma}[p] \neq \emptyset$ and $R_{\Gamma}[\sim p]=\emptyset$ by construction, every rule for $p$ is discarded. Namely, we have $D^{\prime} \vdash-\partial \sim c_{i}$, for all $i$.

Each rule $r_{\sim i}$ is vacuously applicable. Hence, in order to have $-\partial \sim c_{i}$, there must exist a rule $r_{i j}$ that is applicable. Therefore, for each $i$ there is at least one $j$ such that $+\partial a_{j}^{i}$.

We build an interpretation $I$ as follows ${ }^{6}$ :

$$
I\left(a_{j}^{i}\right)=1 \text { iff } D \vdash+\partial a_{j}^{i} .
$$

Since for each $1 \leq i \leq n$, we have $I\left(a_{j}^{i}\right)=1$ for at least one $j$, then also $I \vDash C_{i}$ for all $i$, and we conclude that $I \vDash \Gamma$.

In addition, Theorem 11 specifies that there are situations where it is not possible to revise a literal by modifying only the superiority relation. For instance, if we take a tautological 3-SAT formula, the $\Gamma$-transformation generates a theory that cannot be revised by changing the superiority relation only. We can thus formulate the following result.

Corollary 12 There are theories and literals for which a revision by modifying only the superiority relation is not possible.

This result will be better illustrated by proposing some example-theories at the end of the next section.

A final remark. Previous work for this paper tried to find conditions under which the revision process would be possible and computationally feasible. More proof tags were

${ }^{6}$ We use the standard notation where $I(a)=1$ iff $a$ is evaluated to True in $I$, and $I(a)=0$ otherwise. 
defined with the purpose of identifying conditions that guarantee the success of the revision operation. A revision study based on proof tags has the advantage of the computational efficiency of computing such proof tags. (Remember that the calculus of the extension is polynomial in the size of a theory, and it remains so even with such proof tags.) Unfortunately, even when it is possible to derive conditions for a given literal (in the form of positive/negative proof tags), the revision process may involve some of its antecedents for which we must compute whether are tautologies as well.

Corollary 13 Given any theory $D$, the problem to determine whether a literal $p$ is >$R$-tautological is NP-complete.

Proof. By reduction to the DSC problem.

This means that the revision process generally remains hard. A brief discussion supported by Examples 7 to 8 is proposed at the end of the following section.

\section{Revision in legal domain}

Regardless that we proved that the problem is in general difficult, the proof tags presented in Section 3 are linear to compute in the size of the theory. (The size of a defeasible theory is obtained by counting the number of distinct literals and labels for rules.) This gives us the opportunity to identify cases when: (1) A revision operator may effectively change the theory, (2) the cost of deciding that is (at worst) polynomial. For instance, if there are no chains supporting a given literal $p$, or again if $p$ is $\partial$-unreachable, the formal results proved in Section 3 tell us that no revision on the superiority relation is possible. On the contrary, if $p$ is $\partial$-reachable it is most likely that some revisions are possible.

Similarly to what we did in Section 2, we now motivate the types of changes we study by appealing to the legal domain. When two lawyers dispute a case, there are four situations in which each of them can be if they revise the superiority relation employed by the other one.

(a) The revision process supports the argument of reasonable doubt. Initially, someone proves that the rules imply a given conclusion. The preference can be revised so that that is no longer the case, showing that the conclusion was not beyond reasonable doubt.

(b) The revision process beats the argument of beyond reasonable doubt. Initially, someone proves that a given conclusion does not follow from the rules. However, if the preference is revised the conclusion then follows.

(c) The revision process supports the argument of proof of innocence/guilt. Someone proves that the rules imply a given conclusion. If the preference is revised, then we can derive that the opposite holds.

(d) The revision process cannot support a given conclusion.

Revising a defeasible theory by changing only the priority among its rules means studying how an hypothetical revision operator works in the three cases reported below: 
(1) How to obtain $-\partial p$, starting from $+\partial p$ (contraction).

(2) How to obtain $+\partial \sim p$, starting from $+\partial p$ (revision).

(3) How to obtain $+\partial p$, starting from $-\partial p$ (expansion).

We name these three revisions canonical. In Section 7, we analyse in depth the relationships between the canonical cases and belief revision operations.

Here, we shortly discuss the relationship between the canonical cases and the types of disputes in legal reasoning we introduced above. Situation (a) corresponds to canonical case (1). Situation (b) corresponds to canonical case (3). Situation (c) corresponds to canonical case (2).

Situation (d) includes several contexts that are deemed as sub-cases of the previous ones. In particular, it encompasses those configurations that cannot be effectively revised by the basic operations specified above, namely:

- In the first canonical case, when literal $p$ is $>-R$-tautological (by Definition 7);

- In the second canonical case, when literal $p$ is $>-R$-tautological, or a reasoning chain supporting the complementary does not exist (i.e., condition $-\Sigma \sim p$ holds;

- In the third canonical case, when literal $p$ is $\partial$-unreachable (as stated in Proposition 9), or a reasoning chain supporting it does not exist (i.e., condition $-\Sigma p$ holds).

Note that literals provable with tag $+\varphi$ are special cases of tautological literals (cf. Definition 7). As such, this kind of literals leads the revision process to be unsuccessful for the first and the second canonical case. A possible legal scenario is when one of the parties argues in favour of a thesis in a defeasible way and the counter-part cannot discredit it, or cannot exhibit a proof for the opposite, independently of the changes in the superiority relation. The next proposition formally captures the above intuitions.

Proposition 7 Given a consistent defeasible theory $D=(F, R,>)$, if $D \vdash+\varphi p$ for a literal $p$, then there does not exist a theory $D^{\prime}=\left(F, R,>^{\prime}\right)$ such that (1) $D^{\prime} \vdash+\partial \sim p$, or (2) $D^{\prime} \vdash-\partial p$.

Proof. (1) Given any theory, to obtain a defeasible proof of a literal $q$, there must exist at least one reasoning chain supporting $q$, i.e., $+\Sigma q$ must hold. This is in contradiction with Proposition 2 which states that if $+\varphi q$ holds, also $-\Sigma \sim q$ does. (Note that $-\Sigma l$ implies $-\partial l$, for any literal $l$ : it is trivial to see that if there is no rule with $l$ as conclusion, then proving it is impossible.)

(2) By definition of $+\varphi p$, there exists a reasoning chain that defeasibly proves $p$ made of elements such that there does not exist any (applicable) rule for the opposite. That being the case, no attack to this chain is possible, and condition (2.3.1) of $+\partial$ always holds for each rule of this chain.

Example 7. Consider $D=(\emptyset, R, \emptyset)$, with $R=\{$ 


$$
\begin{aligned}
\Rightarrow_{r_{1}} \quad a \Rightarrow_{r_{2}} \quad b \quad \Rightarrow_{r_{3}} \quad p \\
\Rightarrow_{r_{4}} \quad c \Rightarrow_{r_{5}} \quad q \\
\quad d \Rightarrow_{r_{6}} \neg q \\
\Rightarrow_{r_{7}} \quad e \Rightarrow_{r_{8}} \quad w \\
\Rightarrow_{r_{9}} f \quad \Rightarrow_{r_{11}} g \\
\left.\Rightarrow_{r_{10}} \neg f \Rightarrow_{r_{12}} \neg e\right\} .
\end{aligned}
$$

It is not possible to contract $p$ : in fact, there are no rules for $\neg p$, and neither for $\neg a$ or $\neg b$ (being $a$ and $b$ the only two literals of the only chain ' $r_{1}-r_{2}-r_{3}$ ' supporting, and proving, $p$ ). It is not possible to contract $q: q$ depends on $d$ and there are no rules for $d$ nor $d$ is one of the facts. Lastly, it is not possible to contract $w$. The only possible attack would be on $e$, but $\neg e$ is $\partial$-unreachable.

Example 8. Consider $D=(\emptyset, R, \emptyset)$, with $R=\{$

$$
\begin{aligned}
& a \Rightarrow_{r_{1}} \quad p \\
& \Rightarrow_{r_{2}} \quad b \\
& \Rightarrow_{r_{3}} \neg b \Rightarrow_{r_{4}} \quad c \\
& \left.b, c \Rightarrow_{r_{5}} p\right\} .
\end{aligned}
$$

We cannot revise for $p$. To do successfully so, we would have to change the set of facts (indeed $p$ is $\partial$-reachable due to $r_{1}$ ). We cannot either operate on the provability of $b$ and $\neg b$, which would lead to an inconsistent theory.

Example 9. Consider $D=(\emptyset, R, \emptyset)$, with $R=\{$

$$
\begin{aligned}
& \Rightarrow_{r_{1}} a \Rightarrow_{r_{2}} \quad p \\
& \quad \Rightarrow_{r_{3}} \quad b \quad \Rightarrow_{r_{4}} \quad q \\
& \Rightarrow_{r_{5}} c \\
& \Rightarrow_{r_{6}} \neg c \Rightarrow_{r_{7}} \quad d \\
& \\
& \left.\quad c, d \Rightarrow_{r_{8}} \neg q\right\} .
\end{aligned}
$$

It is not possible to expand for $\neg p$ given that there are no rule for such a literal. We cannot expand for $\neg q$ either given its $\partial$-unreachability.

The theories proposed throughout the previous examples are rather simple (they are all made of a single-literal set of antecedents) but enlighten the underlying problem: the provability/discardability of a given literal may depend on deciding whether any of its premises (or the opposite of one of them) is a tautology, and this is a hard problem in general. More details regarding what a tautology is in a preference-changing setting will be given in the next section.

In the rest of the section, we describe the three types of revision of preferences. For each case we identify the conditions under which such revisions are possible. Therefore, all revision cases studied below will consider $\partial$-reachable literals, assuming that the underlying theories are consistent. Notice that the problem of deciding whether a literal is $\partial$-unreachable is polynomial, given that it is possible to determine when a literal 
is $\partial$-(un)reachable by simply traversing the dependency graph, as proposed in [24]. Furthermore, the proofs tags previously introduced can be computed in polynomial time REF.

The three canonical cases cover the situations where: we pass from a theory proving $p$ to a theory refuting $p$ (without necessarily proving the opposite, $\sim p$ ); we pass from a theory refuting $p$ to a theory proving $p$; and from a theory proving $p$ to a theory proving $\sim p$ (and, thus, refuting $p$ ). Notice that these three cases are the only ones meaningful involving provable and refutable literals. In Section 7, we shall argue that: The first canonical case can be understood as contraction expansion, the second canonical case is a revision, while the third one is expansion of the AGM belief revision framework. Combinatorially, one could consider another case, where $p$ is refuted and we want to obtain a theory where we refute $\sim p$. However, the meaning of this operation is not clear to us, and it is partially subsumed by our third canonical case (given that $+\partial p$ implies $-\partial \sim p)$.

We are now ready to go onto the systematic analysis of the combinations arising from the above defined model. We list the cases by tagging each macroscopic case by the name Canonical case and the combinations depending upon the analytical schema introduced above by the name Instance. The instances show the combination of proof tags where a canonical revision is possible, as well as how to operate on the theory to perform the revision. Where necessary, a general reasoning chain supporting a literal $p$ will be denoted as $P_{p}$.

\subsection{First canonical case: from $+\partial p$ to $-\partial p$}

The first canonical case deals with a situation where there is some arguments proving a claim and we need to change the superiority in order to disprove it. (As said, we want to contract the theory by $p$.)

For the reader to better understand which conditions (in terms of proof tags) hold in the various cases, we report Figure 2 which gives a tree-based graphical representation of all analysed instances. Each brunch represents the proof conditions holding up to that point. A son is tagged with the new condition and inherits all the conditions of its fathers.

For instance, the leftmost leaf labeled with $+\sigma \sim p$ represents the instance where the conditions that hold are: $+\sigma \sim p,+\omega \sim p,-\varphi p$ and $+\Sigma \sim p$. The scheme will be reprised also in the two remaining canonical cases, with the appropriate graphical modifications for the particular case.

Instance $-\Sigma \sim p \wedge+\partial p$ : this case is not reported in Figure 2, but nonetheless it represents a case worth considering. This means there is no supporting chains for $\sim p$, so we cannot operate on them. Given $-\varphi p$, there exists at least one literal in a chain proving $p$, viz. $a$, such that $+\Sigma \sim a$. premises that could be defeated by a rule leading to the opposite conclusion. Thus, in order to obtain $-\partial p$, we have to revise the theory allowing at least one of such rules to be able to fire (to defeat, or at least to have the same power of a rule which actually proves one of the antecedents in the chain supporting $p$ ).

Instance $+\omega \sim p \wedge+\sigma \sim p$ : as stated in Proposition 3, this branch represents an impossible case for any consistent defeasible theory. 


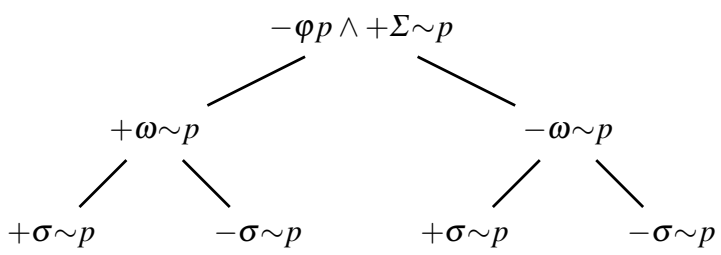

Fig. 2: First canonical case: From $+\partial p$ to $-\partial p$.

Instance $+\omega \sim p \wedge-\sigma \sim p$ : by the straightforward implication of Proposition 3, the chain supporting $\sim p$ fails on the last proof step, defeated by priorities for rules which defeasibly prove $p$. Thus, we have only to erase these priorities.

Instance $-\omega \sim p \wedge+\sigma \sim p$ : since a chain $P_{\sim p}$ exists, and is never defeated (condition $-\omega \sim p$ only illustrates that such a chain fails before the last proof step), a revision process does not have to operate on a chain supporting $p$. We have to strengthen $P_{\sim p}$ by changing many priorities in order to let a rule in $P_{\sim p}$ obtain at least the same strength of such a rule in $P_{p}$. In this process, we do not remove any priority among elements in $P_{p}$, but only add priorities to let a rule in $P_{\sim p}$ win.

Instance $-\omega \sim p \wedge-\sigma \sim p$ : the reasoning chain $P_{\sim p}$ supporting $\sim p$ is defeated, but not necessarily by a chain $P_{p}$ proving $p$. The case is analogous to the aforementioned instance, but (1) we probably have to act not only on $P_{\sim p}$, but also on $P_{p}$, and (2) not only introduce priorities, but erase or invert them. This case represents the most generic situation, where less information is given: a revision is possible, but we do not know $a$ priori where to change the theory.

\subsection{Second canonical case: from $+\partial p$ to $+\partial \sim p$}

By following the cases depicted in Figure 2, we explain how a revision operator should work by changing the root label to " $+\partial p$ to $+\partial \sim p$ " and starting from the same premises $(-\varphi p \wedge+\Sigma \sim p)$. Once more, our revision tree does not take into account tags $\pm \varphi$ for the same reasons explained at the beginning of Section 4.

Instance $+\omega \sim p \wedge+\sigma \sim p$ : as stated in Proposition 3 this branch represents an impossible case for any consistent defeasible theory.

Instance $+\omega \sim p \wedge-\sigma \sim p$ : Proposition 3 states that the chain supporting $\sim p$ is defeated on the last proof step. This, combined with $-\sigma \sim p$, implies that the last step is defeated by a priority for the rule which defeasibly proves $p$. In fact, there may exist more than one defeated chain for $\sim p$ on the last step, as well as more than one chain which proves $p$. We propose two different approaches. We name $P$ the set of chains defeasibly proving $p, P_{l s} \subseteq P$ the set of chains that defeasibly prove $p$ for which there is a priority that applies at the last proof step (against a chain that proves $\sim p$ ), and $N$ the set of chains for which the premises hold:

1. We choose a chain in $N$. We invert the priority for every chain in $P_{l s}$ that wins at the last proof step. We introduce a new priority for making it win against any remaining chain in $P$. 
2. In this approach we have two neatly distinguished cases:

(a) $\left|P_{l s}\right|>|N|$ : for every chain in $N$ we invert the priorities on the last proof step. For every remaining chain in $P$, we add a priority between the defeasible rule used in the last proof step of a chain in $N$ and the rule used in the last proof step of a chain in $P$ (possibly different for each chain in $N$ ) such that the chain in $P$ is defeated.

(b) $|N|>\left|P_{l s}\right|$ : we choose a number $\left|P_{l s}\right|$ of chains in $N$ and invert the priority on the step where they are defeated. If at the end of this step there are still chains in $P$ that defeasibly prove $p$, we go on with the method used for the case 2(a), focusing on the subset of chains in $N$ modified during the first step.

These two approaches rely on different underlying ideas. In the first case we want a unique winning chain. This makes the revision procedure faster than the second method, since we do not have to choose a different chain to manipulate every time. Moreover, the number of changes made with the first approach is equal to that of the second one in the worst case scenario (in general, it revises the theory with the minimum number of changes).

The strength of the second method relies on the concept of team defeater: we do not give power to a single element, but to a team of rules. In the first method the entire revision process must be repeated once the winning chain is defeated, while in the second method if one of the winning chains is defeated, we have only to apply the revision process on it, and not on all the other winning chains.

Let us consider the following example:

$$
\begin{array}{cc}
\Rightarrow_{r_{1}} p & \Rightarrow_{r_{2}} p \\
\vee & \vee \\
\Rightarrow_{r_{3}} \neg p & \Rightarrow_{r_{4}} \neg p
\end{array}
$$

If the chain for $\neg p$ with rule $r_{4}$ is chosen as the winning chain, the first approach would give $\left\{r_{1}>r_{3}, r_{4}>r_{1}, r_{4}>r_{2}\right\}$ as an output, erasing one priority and introducing two, while the second approach would generate the following priority set: $\left\{r_{3}>r_{1}, r_{4}>\right.$ $\left.r_{2}\right\}$, erasing two priorities, and introducing two. If a new rule $r_{i}$ defeats $r_{4}$, it is easy to see that in the first case we have to entirely revise the theory (for example, let $r_{3}$ win against $r_{1}$ and $r_{2}$ ), while in the second case we have only to introduce $r_{3}>r_{2}$.

Instance $-\omega \sim p \wedge+\sigma \sim p$ : there exists an undefeated chain supporting $\sim p$. To revise the theory, we have to choose one of them and, starting from $\sim p$, go back in the chain to the ambiguity point (where $P(i)=+\partial p_{i} \wedge P(i+1)=-\partial p_{i+1}$ holds), and strengthen the chain by adding a priority where a rule leading to an antecedent in the chain for $\sim p$ and a rule for the opposite have the same strength.

Instance $-\omega \sim p \wedge-\sigma \sim p$ : every chain supporting $\sim p$ is defeated at least one time. A plausible solution could be to go back in the chain searching for the point where $P(i)=+\sigma p_{i}$ and $P(i+1)=-\sigma p_{i+1}$. But this is not enough to guarantee the chain to win. Let us consider the following example. 
Example 10. Let $D$ be a theory having the following rules:

$$
\begin{array}{cc}
+\partial /-\partial & +\sigma /-\sigma \\
\Rightarrow_{r_{1}} \quad a \Rightarrow_{r_{2}} b \stackrel{r_{r_{3}}}{\wedge^{\prime}} \quad c \Rightarrow_{r_{4}} p \\
\Rightarrow_{r_{5}} \neg a \quad \stackrel{\Rightarrow_{r_{6}}}{\neg} \neg c
\end{array}
$$

It is easy to see that the sole condition of $r_{3}$ winning over $r_{6}$ is not sufficient: we have also to introduce a priority between $r_{1}$ and $r_{5}$. Thus, we have to act exactly as in the aforementioned case, with the solely difference that every time a rule in the chain supporting $\sim p$ is defeated, the priority rule has to be inverted.

\subsection{Third canonical case: from $-\partial p$ to $+\partial p$}

For a proper analysis of this case, condition $-\partial \sim p$ must hold since otherwise the case is analogous of the previous revision from $+\partial q$ to $+\partial \sim q$. Also, we do not take into consideration the case where $-\Sigma p$ holds: if there are no chains leading to $p$, then no revision to obtain $+\partial p$ is possible. The cases studied in this subsection are reported in Figure 3.

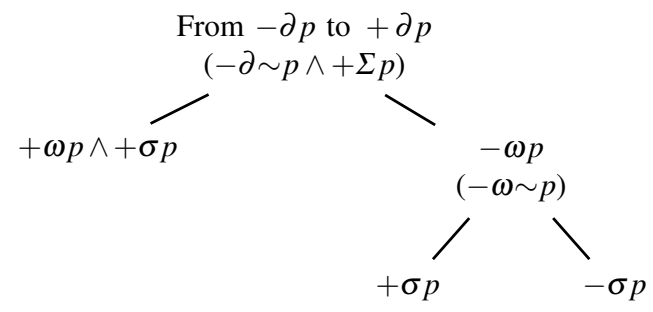

Fig. 3: From $-\partial p$ to $+\partial p$ : revision cases.

Notice that $+\omega p$ and $-\sigma p$ cannot hold at the same time: if all the premises for $p$ are proved, then the chain fails on the last step, i.e., it has to be defeated by a firing rule for $\sim p$. This would defeasibly prove $\sim p$, but this cannot happen since we have stated that $-\partial \sim p$ holds. Furthermore, $-\omega p$ implies that $-\omega \sim p$ holds as well, since if it is not the case, we would have either $+\omega p$, or $+\partial \sim p$, which are both against the hypothesis.

Instance $+\omega p \wedge+\sigma p$ : we choose one of the chains where condition $+\omega p \wedge+\sigma p$ holds, and introduce as many priorities as the number of chains where $+\omega \sim p$ holds.

Instance $-\omega p \wedge+\sigma p$ : this case is analogous to instance $-\omega \sim p \wedge+\sigma \sim p$ of canonical case from $+\partial p$ to $+\partial \sim p$.

Instance $-\omega p \wedge-\sigma p$ : this case is analogous to instance $-\omega \sim p \wedge-\sigma \sim p$ of canonical case from $+\partial p$ to $+\partial \sim p$. 
We remark that conditions $\pm \sigma \sim p$ are not useful for the revision process, since they do not give information whether the changes will affect chains for $\sim p$, or not. Example 11 shows that, even if $+\sigma \neg p$ holds, two distinct revisions exist: the first involves the chain for $\neg p$ (introducing $r_{1}>r_{3}$ ), the second does not (introducing $r_{5}>r_{6}$ ).

Example 11. Let $D$ be a theory having the following rules:

$$
\begin{aligned}
& \Rightarrow_{r_{1}} a \Rightarrow_{r_{2}} p \\
& \Rightarrow_{r_{3}} \neg a \Rightarrow_{r_{4}} \neg p \\
& \Rightarrow_{r_{5}} b \Rightarrow_{r_{6}} p \\
& \Rightarrow_{r_{6}} \neg b
\end{aligned}
$$

An analogous situation is proposed for $-\sigma \sim p$ in Example 12 .

Example 12. Let $D$ be a theory having the following rules:

$$
\begin{aligned}
\Rightarrow_{r_{1}} a & \Rightarrow r_{r_{2}} p \\
\Rightarrow r_{r_{3}} \neg a & \Rightarrow r_{r_{4}} \quad b \Rightarrow r_{r_{5}} \neg p \\
& \wedge \\
& \Rightarrow_{r_{6}} \neg b \\
\Rightarrow_{r_{7}} c & \Rightarrow_{r_{8}} p \\
\Rightarrow_{r_{9}} \neg c &
\end{aligned}
$$

In here, two revisions exist: one introducing $r_{1}>r_{3}$, and the other one which introduces $r_{7}>r_{9}$.

Notice that in all the canonical cases, the revision mechanism guarantees that no cycle can be introduced. We can formulate this result, which is a straightforward consequence of the case analysis presented here.

Theorem 14 Revising a superiority relation generates a superiority relation.

\section{Preference revision based on canonical cases}

In this section we investigate conditions under which we can revise a defeasible theory by only changing the preference relation. In particular, we propose algorithms corresponding to the three canonical cases presented in the previous section and their conditions of applicability. The algorithms are not intended to be optimal in the sense of finding minimal revisions ${ }^{7}$, but they just provide a constructive mechanism to guarantee that a revision exists.

Proposition 7 gives us a condition under which we know some revisions are not possible. Specifically, if $+\varphi p$ holds, then it is not possible to have revisions for $+\partial \sim p$,

\footnotetext{
${ }^{7}$ It is possible to give alternative definitions of minimal revision. For example, one notion could be on the cardinality of instances of the superiority relation we have to modify, while another one is to consider minimality with respect to the conclusions derived from a theory.
} 
or $-\partial p$. Thus, we have a first restriction for the canonical case from $+\partial p$ to $-\partial p$ (and also from $+\partial \sim p$ to $+\partial p$ ).

Before embarking on the description of algorithms for revisions corresponding to the canonical cases, we have to identify further conditions under which a revision is possible. To this aim we have to introduce some preliminary definitions. We start by defining when a literal depends on another literal.

Definition 15 Let $a$ and $b$ be two literals in a defeasible theory $T$. Then $a$ depends on $b$ if, and only if (1) $b=a$ or (2) $\forall r \in R[a]$, either (2.1) $b \in A(r)$, or (2.2) $\exists c$ such that $c \in A(r)$, and $c$ depends on $b$.

The following result shows that a defeasibly proved literal implies also the provability of all literals it depends on. In other words, the property of dependency given above propagates backwards the defeasible provability of literals.

First, we identify

Proposition 8 Given a defeasible theory $T$, if $T \vdash+\partial p$ and $p$ depends on $q$, then $T \vdash$ $+\partial q$.

Proof. Case (1) of Definition 15 trivially holds, as $p$ and $q$ are the same literal. For the other cases, the proof is by induction on the degree of dependency between literals. A literal $a$ depends on $b$ with degree 1 if $a$ depends on $b$ and there is a rule for $a$ where $b$ belongs to the antecedents of the rule. A literal $a$ depends on $b$ with degree $n+1$ if $a$ depends on $b$ and there is a literal $c$ such that $a$ depends on $c$ with degree 1 and $c$ depends on $b$ with degree $n$.

For the inductive base (i.e., $p$ depends on $q$ with degree 1 ), $+\partial p$ means that there is a rule such that for every element $a$ in the antecedents, we have $T \vdash+\partial a$. Thus, $T \vdash+\partial q$.

For the inductive step, suppose that the property holds up to degree $n$ and $p$ depends on $q$ with degree $n+1$. By definition, there is a literal $c$ such that $p$ depends on $c$ with degree 1, thus $T \vdash+\partial c$ (given $T \vdash+\partial p$ by hypothesis) and $c$ depends on $q$ with degree $n$. Thus, by inductive hypothesis, $T \vdash+\partial q$.

The idea of the next definition is to identify literals only depending on contradictions. For example, consider the theory with the following rule

$$
a, \neg a, b \Rightarrow_{r} p .
$$

To prove $+\partial p$, we need both $+\partial a$ and $+\partial \neg a$ and this is possible only if the theory is inconsistent. However, we have also to cater for situations where the dependency is not direct, for example, theories like

$$
a \Rightarrow_{r_{1}} b \quad \neg a, b \Rightarrow_{r_{2}} p .
$$

Definition 16 A literal $p$ is $\partial$-unreachable if, and only if $\forall r \in R[p]$, either (1.) $\exists l, \exists a, b \in$ $A(r)$ such that (1.1) a depends on l, and (1.2) $b$ depends on $\sim l$, or (2.) $\exists d \in A(r)$ such that d is d-unreachable. 
The result below formalises the relationship between $\partial$-unreachable literals and inconsistent theories.

Proposition 9 Given a defeasible theory $T$, let $p$ be a $\partial$-unreachable literal. If $T \vdash$ $+\partial p$, then $T$ is inconsistent.

Proof. The proof is by induction on the number of $\partial$-unreachable literals in a derivation.

In the base case, $p$ is the only $\partial$-unreachable literal in its derivation. Given that $T \vdash+\partial p$, there is a rule for $p$ such that all its antecedents are provable. By definition of $\partial$-unreachable literal, for every rule for $p$ there are two literals $a$ and $b$ in the antecedents of the rule depending on a literal $l$ and its complement, respectively. Thus we have both $+\partial a$ and $+\partial b$. Then by Proposition 8, we have $T \vdash+\partial l$ and $T \vdash+\partial \sim l$, thus $T$ is inconsistent.

For the inductive step, we assume that the property holds up to $n \partial$-unreachable literals, and $p$ is the $(n+1)^{\text {th }} \partial$-unreachable literal. Beside the case we examined in the inductive base, we have to consider that the antecedent of a rule contains a $\partial$-unreachable literal $d$ and $T \vdash+\partial d$. Thus, $d$ falls under the inductive hypothesis, therefore $T$ is inconsistent.

The following proposition states that, if there is at least one supporting chain for a literal that is not $\partial$-unreachable, it is always possible to revise the theory to let the literal be defeasibly proven. In other words, the problem of modifying the superiority relation of a defeasible theory in order to pass from $-\partial p$ to $+\partial p$ (or from $+\partial \sim p$ to $+\partial p$ ) has always a solution, provided that the condition $+\Sigma p$ holds.

Proposition 10 Given a consistent defeasible theory $T=(F, R, \sup )$ such that $T \vdash$ $+\Sigma p$ and $p$ is not $\partial$-unreachable, there exists a theory $T^{\prime}=\left(F, R, s u p^{\prime}\right)$ such that $T^{\prime} \vdash+\partial p$.

Proof. Proposition 9 shows that a $\partial$-unreachable literal is provable only when the theory is inconsistent, which is against the hypothesis of the proposition.

Suppose that $T \vdash+\Sigma p$ for a defeasible theory $T$. Then, there is at least one reasoning chain $C$ supporting $p$. Among all the possible superiority relations based on $F$ and $R$, there is a superiority relation $\sup ^{\prime}$ where every rule $r: A \Rightarrow c$ in $C$ is superior to any rule for $\sim c$, and then no rule is superior to $r$. Thus, the theory $T^{\prime}=\left(F, R, s u p^{\prime}\right)$ is such that $T^{\prime} \vdash+\partial p$.

To illustrate why the two conditions of Proposition 10 are required to guarantee that the canonical case whose outcome is that $+\partial p$ after the revision operation, let us consider a theory with the following rules:

$$
\begin{aligned}
& \Rightarrow_{r_{1}} a \\
& \Rightarrow_{r_{2}} \neg a \\
& \quad a, \neg a \Rightarrow_{r_{3}} p
\end{aligned}
$$

In this case we have both $+\Sigma a$ and $+\Sigma \neg a$, thus we can build a reasoning chain to $p$, but $p$ itself is $\partial$-unreachable, given that it depends on a contradiction.

We now shift the focus on conditions on the superiority relation. First of all, we introduce some notation. 
Definition 17 For a given literal $p$ :

$$
\begin{aligned}
& \sup _{\text {LAST }}(p)=\left\{(s, t) \mid s \in R_{s d}[p], t \in R_{s d}[\sim p] \text { and }+\omega \sim p\right\} \\
& \widetilde{\sup }_{L A S T}(p)=\left\{(t, s) \mid(s, t) \in \sup _{L A S T}(p)\right\} .
\end{aligned}
$$

The sets $\sup _{\text {LAST }}(p)$ and $\widetilde{\sup }_{\text {LAST }}(p)$ allow us to find out all the rules leading to the opposite of literal $p$ in a chain defeated on the last proof step.

Lemma 1. Given a defeasible theory $T=(F, R$, sup $)$, if $T \vdash+\omega p$ for a literal $p$, then the superiority relation sup' given by

$$
\sup ^{\prime}=\left(\sup \cup \sup _{\text {LAST }}(p)\right) \backslash \widetilde{\sup }_{\text {LAST }}(p)
$$

is such that $T^{\prime} \vdash+\partial p$, where $T^{\prime}=\left(F, R, s u p^{\prime}\right)$.

Proof. The thesis trivially holds by definition of the tag $+\omega$ and by Definition 17 .

The meaning of the above Lemma is that given a set of rules for a literal $p$, if these rules are applicable (all the elements of the antecedent of the rules are defeasible provable), then specifying that these rules are stronger than the rules for the opposite conclusion, i.e., $\sim p$, guarantees that we can defeasibly prove $p$.

Lemma 2. Given a theory $T=(F, R$, sup $)$, if $T \vdash+\partial p$ and $T \vdash+\omega \sim p$ for a literal $p$, then the superiority relation sup' given by

$$
\sup ^{\prime}=\sup \backslash \widetilde{\sup }_{L A S T}(p)
$$

is such that $T^{\prime} \vdash-\partial p$, where $T^{\prime}=\left(F, R, \sup ^{\prime}\right)$.

Proof. The thesis trivially holds by definition of the tag $+\omega$ and the definition of $\sup _{\text {LAST }}$. Relation sup' is clearly acyclic as the initial superiority relation sup is acyclic and $\sup ^{\prime}$ is obtained only erasing tuples.

The meaning of Lemma 2 is similar to that of Lemma 1. This time, ensuring that the (applicable) rules for $p$ have the same strength of those for $\sim p$ prevents us from defeasibly deriving $p$.

In the following subsections, we present techniques to algorithmically compute priority revisions with respect to the three canonical cases. After each algorithm, we propose technical explanations on how they work, followed by their computational results, and running examples.

\subsection{Algorithm for the first canonical case}

We report below the algorithm for the first canonical case, i.e. from $-\partial p$ to $+\partial p$ (Algorithm 1). 
Input: a defeasible theory $T=(F, R, \sup )$ and a literal $p$ such that $T \vdash-\partial p$, $T \vdash-\partial \sim p$, and $T \vdash+\Sigma p$.

Output: a defeasible theory $T^{\prime}=\left(F, R, s u p^{\prime}\right)$ such that $T^{\prime} \vdash+\partial p$.

1 Let $Q$ be an empty set;

2 if $+\omega p$ then

\begin{tabular}{l|l}
3 & $\sup \longleftarrow\left(\sup \cup \sup p_{L A S T}(p)\right) \backslash \widetilde{\sup }_{L A S T}(p) ;$ \\
4 & return $(F, R$, sup $) ;$
\end{tabular}

5 end if

$6 \longleftarrow\{p\}$;

7 repeat

$8 \quad$ Calculate $R[Q]=\left\{r \in R_{s d}[q] \mid q \in Q\right\} ;$

9 foreach $x \in A(R[Q])$ do

$10 \mid$ if $+\omega \sim x$ then

11

12

13

14

15

16

17

18

19

20

21

22 until $A(R[Q])=\emptyset$;

$\mid \sup \longleftarrow\left(\sup \cup \sup p_{L A S T}(x)\right) \backslash \widetilde{\sup }_{L A S T}(x) ;$

end if

if $+\partial x$ then

end if

end foreach

if $+\partial p$ then

| return $(F, R$, sup $)$;

else

| $Q \longleftarrow A(R[Q])$;

end if

Algorithm 1: from $-\partial p$ to $+\partial p$

We now examine how Algorithm 1 works. Algorithm 1 can be seen as a doublephase procedure. In the preliminary phase, it checks if there is at least one chain for $p$ such that all its antecedents are defeasibly proved, but the chain does not win at the last proof step (if condition guard at line 2). In this case, the algorithm simply adds a preference between the rule leading to $p$ in such chains and all the rules leading to $\sim p$ (line 3). It then returns the theory with the same sets of facts and rules along with the new preference relation sup (line 4).

The second phase of the algorithm takes place if condition at line $\mathbf{2}$ does not hold. This means that for every chain supporting $p$, at least one rule for an antecedent is defeated by a rule for its opposite, or have the same strength. The repeat cycle at lines 7 - 22 takes care of backtracking one step at a time in these chains to resolve the possible conflicts:

1. the support set $Q$ contains all the literals analysed in the backtracking steps (the set is initialised with literal $p$ at line $\mathbf{6}$, and iteratively computed at line 8);

2. using the set $R[Q]$ (containing all the rules leading to every literal in $Q$ ), the algorithm calculates all the possible antecedents for a literal in $Q$ (line 9); 
3. if for one of those antecedents there is an applicable rule for the opposite (line 10), the preference relation is modified in a way similar to the preliminary phase (line 11);

4. if this operation makes the current literal defeasibly proved, then backtracking in its chain will bring no more changes, and therefore it can be erased from the set (line 14);

5. if the condition of provability for $p$ has not yet been achieved, then the algorithm has to step back once more in the chains, and therefore the remaining literals represent the elements for the next step (line 20). Otherwise, the algorithm terminates returning the revised theory.

Notice that after the execution of Algorithm 1, condition $+\Sigma p$ still holds. In fact, the only way to obtain $-\Sigma p$ is to erase derivation rules from $R$, but this cannot happen since Algorithm 1 only modifies the superiority relation sup. In other words, the revision process made by the algorithm does not affect the rules set nor facts, but acts only on the superiority relation.

Theorem 18 Algorithm 1 is correct, complete and terminates.

Proof. Algorithm 1 terminates in a finite number of steps. The algorithm can be divided indeed in two distinct parts, which are executed in a mutual exclusive way depending on whether the condition $+\omega p$ is true or not at the beginning of the execution.

In the first case, the part of the algorithm to consider is from lines $\mathbf{2}$ to $\mathbf{5}$. Since the corresponding if cycle manipulates finite sets of literals, and the union of finite sets is fully decidable, termination is guaranteed. The correctness of the solution returned by step 4 follows by Lemma 1.

In the second case, condition $+\omega p$ is not met at the beginning of the algorithm, and the execution flow goes directly to line $\mathbf{6}$. Steps from $\mathbf{7}$ to $\mathbf{2 2}$ are executed at most as many times as the length of the longest finite chain leading to $p$. Since the length of the longest finite chain is at most the number of letters in $T$, and $T$ has a finite signature, then the outermost repeat cycle (lines 7-22) terminates provided that condition in line 17 is eventually satisfied. Suppose by contradiction that the revision operated at steps 10-15 does not defeasibly prove $p$ at any step. Since in the inner cycle, step 10 checks if a literal (that is the opposite of an antecedent of a rule used in a chain leading to $p$ ) wins, and step 11 changes this condition, the only possibility for the inner cycle not to derive $+\partial p$ is to backtrack in the outer cycle until the first literal of the longest finite chain leading to $p$ without passing the test of line $\mathbf{1 0}$ (since operation at line $\mathbf{1 4}$ removes only elements that are now defeasibly provable, it is no more necessary to continue checking their supporting chain). In this situation, again, every chain supporting $p$ wins. A contradiction.

Notice that the algorithm is trivially complete given that it always terminates, and the problem always has a solution by Proposition 10.

We now exhibit the computational complexity of Algorithm 1. To do so we employ the notion of dimension of a defeasible theory, that accounts for the total number of occurrences of the literals occurring in proper axioms. 
Theorem 19 The computational complexity of Algorithm 1 is $O\left(d^{3}\right)$, where $d$ is the dimension of the theory.

Proof. Assume that the initial defeasible theory $T=(F, R$, sup $)$ has dimension $d$. The length of the longest chain analysed by Algorithm 1 is $l \leq d$. In the worst case, when only one chain is processed, this is exactly $d$. The if condition at lines $\mathbf{2 - 5}$ is of order $O(d)+O(d)=O(d)^{8}$. The inner foreach cycle (lines 9-16) is of order $O(d)$. Again, the if condition at lines 17-21 is of order $O(d)+O(d)=O(d)$. As the outer repeat cycle is iterated $O(d)$ times, its complexity is of order $O\left(d^{3}\right)$. Therefore, Algorithm 1 has an overall complexity of $O(d)+O\left(d^{3}\right)=O\left(d^{3}\right)$.

Examples 13, and 14 show how Algorithm 1 works with two different theories in order to defeasibly derive $+\partial p$.

Example 13. Let $T$ be a theory having the following rules:

$$
\begin{aligned}
& \Rightarrow_{r_{1}} a \Rightarrow_{r_{2}} p \\
& \Rightarrow_{r_{3}} b \Rightarrow_{r_{4}} \neg p
\end{aligned}
$$

Since $T \vdash+\partial a$, the if condition at line $\mathbf{2}$ is satisfied, and Algorithm 1 adds $r_{2}>r_{4}$ to the superiority relation.

Example 14. Let $T$ be a theory having the following rules:

$$
\begin{aligned}
& \Rightarrow_{r_{2}} a \Rightarrow_{r_{3}} b, c \Rightarrow_{r_{1}} p \\
& \wedge \\
& \Rightarrow_{r_{4}} \neg a \\
& \Rightarrow_{r_{5}} \neg b \\
& \Rightarrow_{r_{6}} c \\
& \Rightarrow_{r_{7}} d \Rightarrow_{r_{8}} \neg c \\
& \Rightarrow_{r_{9}} \neg d
\end{aligned}
$$

The if condition at line $\mathbf{2}$ is not satisfied, then iteration of the repeat and foreach cycles starts: set $Q$ is initialised with $\{p\}, R[Q]$ with $\left\{r_{1}\right\}$, and $A(R[Q])$ with $\{b, c\}$. Analysing the first literal in $A(R[Q])$, i.e., $b$, the if condition at line $\mathbf{1 0}$ is satisfied, so $r_{3}>r_{5}$ is added. Since $+\partial a$ does not hold, $b$ still remains in $A(R[Q])$. During the second iteration of the foreach cycle, condition at line $\mathbf{1 0}$ does not hold for $\neg c$, as $-\partial d \Rightarrow-\omega \neg c$. Hence, no operations are needed, and $c$ is removed from $A(R[Q])$ at line 14. The algorithm steps to the next repeat cycle iteration: $Q$ is updated to $\{b\}$, $R[Q]$ to $\left\{r_{3}\right\}$, and $A(R[Q])$ to $\{a\}$. As $+\partial \neg a$ holds, test at line $\mathbf{1 0}$ is true, and algorithm inverts the existing priority relation $r_{4}>r_{2}$. After this last modification, the resulting theory defeasibly proves $p$, and so Algorithm 1 terminates.

\footnotetext{
${ }^{8}$ Notice that verifying the provability of a proof tag is linear in the order of the theory, see [23].
} 


\subsection{Algorithm for the second canonical case}

We now exhibit the algorithm corresponding to the second canonical case, i.e., from $+\partial \sim p$ to $+\partial p$.

Input: a defeasible theory $T=(F, R$, sup $)$ and a literal $p$ such that $T \vdash+\partial \sim p$ and $T \vdash+\Sigma p$.

Output: a defeasible theory $T^{\prime}=\left(F, R, s u p^{\prime}\right)$ such that $T^{\prime} \vdash+\partial p$.

1 Let $Q$ be an empty set;

2 if $+\omega p$ then

$3 \mid \sup \longleftarrow\left(\sup \cup \sup p_{L A S T}(p)\right) \backslash \widetilde{\sup }_{L A S T}(p)$;

4 return $(F, R$, sup $)$;

5 end if

$6 Q \longleftarrow\{p\}$;

7 repeat

$8 \quad$ Calculate $R[Q]=\left\{r \in R_{s d}[q] \mid q \in Q\right\} ;$

$9 \quad$ foreach $x \in A(R[Q])$ do

$10 \mid$ if $+\omega \sim x$ then

$11 \quad \mid \sup \longleftarrow\left(\sup \cup \sup \operatorname{paST}_{L A}(x)\right) \backslash \widetilde{\sup }_{\text {LAST }}(x)$;

12 end if

$13 \quad$ if $+\partial x$ then

14

15

16

17

18

19

20

21

22 until $A(R[Q])=\emptyset$;

23 sup $\longleftarrow\left(\sup \cup \sup p_{L A S T}(p)\right) \backslash \widetilde{\sup }_{L A S T}(p)$;

24 return $(F, R$, sup $)$;

Algorithm 2: from $+\partial \sim p$ to $+\partial p$

Algorithm 2 acts following the same idea exploited by Algorithm 1: in order to obtain a revised theory where $+\partial p$ holds, we can essentially ignore whether in the initial theory $+\partial \sim p$ holds or not, and make the same operations of Algorithm 1. Therefore, also in this case, there must be at least one chain supporting $p$ in the initial theory, i.e., the condition $+\Sigma p$ holds.

In the first canonical case, the application of Algorithm 1 forces every chain supporting $\sim p$ to be defeated by the revision process. It is not, however, self evident whether this also holds for the second canonical case. We can partition the set of all chains which defeasibly prove $\sim p$ in two sets: a chain for $\sim p$ is in the first set if at least one of its elements is the opposite of a literal in a chain supporting $p$, otherwise it is in the second set. Thus, if a chain is in the first set, Theorem 18 proves that it will be eventually defeated according to the procedure at lines 7-22. Finally, if a chain is in the second 
set, it will be defeated by the priority rules on the last derivation step according to the assignment at line $\mathbf{2 3}$.

Theorem 20 Algorithm 2 is correct, complete, and always terminates.

Proof. The proof straightforwardly follows from Theorem 18 and by the above reasoning.

Theorem 21 exhibits the computational complexity of Algorithm 2.

Theorem 21 The computational complexity of Algorithm 2 is $O\left(d^{3}\right)$, where $d$ is the dimension of the theory.

Proof. Algorithm 2 has the same (polynomial) complexity of Algorithm 1, since they differ only for the assignment at line 23, which is of order $d$. Thus, Theorem 19 binds the complexity to $O\left(d^{3}\right)$.

Below we propose an example to show how Algorithm 2 works.

Example 15. Let $T$ be a theory having the following rules:

$$
\begin{aligned}
\Rightarrow_{r_{1}} a & \Rightarrow_{r_{2}} \quad b \Rightarrow_{r_{3}} p \\
\Rightarrow_{r_{4}} c \Rightarrow_{r_{5}} \neg a & \\
& \Rightarrow_{r_{6}} \neg b \Rightarrow_{r_{7}} \neg p \\
& \Rightarrow_{r_{8}} d \Rightarrow r_{r_{9}} \neg p
\end{aligned}
$$

The if condition at line $\mathbf{2}$ is not satisfied, then the iteration of the repeat and foreach cycles starts: set $Q$ is initialised with $\{p\}, R[Q]$ with $\left\{r_{3}\right\}$, and $A(R[Q])$ with $\{b\}$. Analysing literal $b$, the if condition at line $\mathbf{1 0}$ is satisfied, so $r_{2}>r_{6}$ is added. Since $+\partial a$ does not hold, $b$ still remains in $A(R[Q])$. There are no other elements in $A(R[Q])$, thus the algorithm steps to the next repeat cycle iteration: $Q$ is updated to $\{b\}, R[Q]$ to $\left\{r_{2}\right\}$, and $A(R[Q])$ to $\{a\}$. As test at line $\mathbf{1 0}$ is true: $r_{1}>r_{5}$ is added to the priority relation at line 11, and literal $a$ is erased from $A(R[Q])$ since condition at line $\mathbf{1 3}$ is satisfied. $A(R[Q])$ is now empty, and so the repeat cycle ends. Finally, at line 23, preference $r_{3}>r_{9}$ is added to the theory. The resulting theory defeasibly proves $p$, and Algorithm 2 terminates. 


\subsection{Algorithm for the third canonical case}

The last algorithm we propose is the one to compute the revision from $+\partial p$ to $-\partial p$.

Input: a defeasible theory $T=(F, R, \sup )$ and a literal $p$ such that $T \vdash+\partial p$ and $T \vdash-\varphi p$.

Output: a defeasible theory $T^{\prime}=\left(F, R, s u p^{\prime}\right)$ such that $T^{\prime} \vdash-\partial p$.

1 Let $Q$ be an empty set;

2 if $+\omega \sim p$ then

$3 \mid \sup \longleftarrow \sup \backslash \sup _{L A S T}(p)$;

4 return $(F, R$, sup $)$;

5 end if

$6 \longleftarrow\{p\}$;

7 repeat

$8 \quad$ Calculate $R[Q]=\left\{r \in R_{s d}[q] \mid q \in Q \wedge+\partial A(r)\right\}$;

$9 \quad$ foreach $x \in A(R[Q])$ do

$10 \mid$ if $+\omega \sim x$ then

11

12

13

14

15

16

17

$18 \quad Q \longleftarrow A(R[Q])$;

19 until $A(R[Q]) \neq \emptyset$;

$20 \quad Q \longleftarrow\{p\}$;

21 repeat

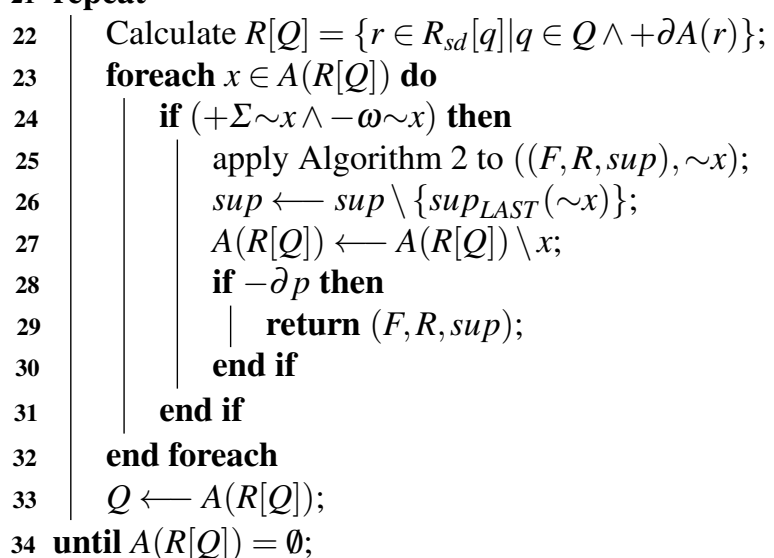

Algorithm 3: from $+\partial p$ to $-\partial p$

A chain defeasibly proves a literal $p$ when every rule, supporting the opposite of a element in such a chain, is either defeated by a priority rule, or not applicable. Hence, 
in order to revise a theory for the third canonical case from $+\partial p$ to $-\partial p$, we have to make these opposite literals applicable by:

1. removing those priority rules that let a chain supporting $p$ win;

2. letting an opposite element to have the same strength of the corresponding one in the chain for $p$, i.e., defeasibly proving all its antecedents.

The first point is performed by Algorithm 3 from line 2 to line 18, considering both the case when the chain has a priority rule on the last proof step (lines 2-5), and when it has priorities on rules for antecedents of $p$ (cycle at lines 7-19).

The second point is performed at lines 20-34: firstly Algorithm 2 is invoked with parameter $\sim p$; after this, assignment at line $\mathbf{2 6}$ makes this literal of the same strength of the corresponding one in a chain for $p$. Notice that if guard at line $\mathbf{2 4}$ checks both $+\Sigma$, and $-\omega$ proof tags since it could happen that the execution of Algorithm 3 changed a literal from $-\omega$ to $+\omega$. Thus, no more changes are needed for this literal.

Theorem 22 Algorithm 3 is correct, complete, and always terminates.

Proof. Since $-\varphi p$ holds, the definition of $-\varphi$ ensures that a rule for the opposite of an element in every chain supporting $p$ exists. By definition of $+\partial p$ the cases when a chain defeasibly proves $p$ are only the ones described in the reasoning above. Therefore, no further cases arise that Algorithm 3 does not takes care of. In all those situations, the above reasoning, Lemma 2 and Theorem 20 guarantee correctness of the solution. Thus, since a solution always exists, Algorithm 3 terminates.

In analogy to the complexity analysis of Algorithm 3, we show here that the problem solved by the herein cited algorithm is applied to the complexity of Algorithm 2.

Theorem 23 The computational complexity of Algorithm 3 is $O\left(d^{5}\right)$, where $d$ is the dimension of the theory.

Proof. Assume that the defeasible theory $T=(F, R, \sup )$ has dimension $d$. Again, the assumptions given in the proof of Theorem 19 for the length of the longest analysed chain hold. Complexity of the if condition at lines 2-5, and of the repeat cycle at lines 7-19 has already been proven in Theorem 19 to be respectively $O(d)$, and $O\left(d^{3}\right)$. Similarly, the repeat cycle at lines $\mathbf{2 1 - 3 4}$ is iterated at most $d^{2}$ times: this reasoning, and Theorem 21 guarantee that its complexity is of order $O\left(d^{5}\right)$. Therefore, Algorithm 3 has an overall complexity of $O(d)+O\left(d^{3}\right)+O\left(d^{5}\right)=O\left(d^{5}\right)$.

As for the two previous algorithms, we now exhibit an example that illustrates the behaviour of the algorithm. 
Example 16. Let $T$ be a theory having the following rules:

$$
\begin{gathered}
\Rightarrow_{r_{1}} \quad a \Rightarrow_{r_{2}} p \\
\quad \Rightarrow_{r_{3}} \neg a \\
b, d \Rightarrow_{r_{4}} p \\
\Rightarrow_{r_{5}} \quad b \\
\Rightarrow_{r_{6}} c \Rightarrow_{r_{7}} \neg b \\
\Rightarrow_{r_{8}} \neg c \\
\Rightarrow_{r_{9}} d \\
\Rightarrow_{r_{10}} e \Rightarrow_{r_{11}} \neg d \\
\Rightarrow_{r_{12}} \neg e
\end{gathered}
$$

The if condition at line $\mathbf{2}$ is not satisfied, then the iteration of the repeat and foreach cycles starts: set $Q$ is initialised with $\{p\}, R[Q]$ with $\left\{r_{2}, r_{4}\right\}$, and $A(R[Q])$ with $\{a, b, d\}$. Analysing the first literal in $A(R[Q])$, i.e., $a$, the if condition at line $\mathbf{1 0}$ is satisfied, so the priority relation $r_{1}>r_{3}$ is removed from the initial theory, as literal $a$ from $A(R[Q])$; $+\partial p$ still holds. In the next two iterations of the foreach cycle, condition at line $\mathbf{1 0}$ holds neither for $\neg b$, nor for $\neg d$, hence no operations are executed. Similarly, this happens in the following two iterations of the repeat cycle, since there are no more rules in a chain for $p$ that could be defeated acting on the superiority relation.

The algorithm sets $Q, R[Q]$, and $A(R[Q])$ to the initial values. The foreach cycle at line $\mathbf{2 3}$ analyses literal $a$ first, but since condition at line $\mathbf{2 4}$ does not hold, no operations are performed during this foreach iteration. In the next iteration, literal $b$ is analysed and, since condition at line $\mathbf{2 4}$ is satisfied, Algorithm 2 is invoked. It returns a superiority relation with two new preferences, $r_{7}>r_{5}$ and $r_{6}>r_{8}$. The last one is then removed and so literal $b$ from $A(R[Q])$, at lines $\mathbf{2 6}$ and $\mathbf{2 7}$, respectively. Again, since condition at line $\mathbf{2 8}$ is not satisfied, the repeat cycle goes to the next iteration, analysing literal $d$, invoking Algorithm 2 which adds $r_{11}>r_{9}$ and $r_{10}>r_{12}$ to the superiority relation. Once more, the last one is removed and so literal $d$ from $A(R[Q])$. Now condition at line $\mathbf{2 8}$ is satisfied, and so Algorithm 3 terminates.

\section{AGM postulates analysis}

The aim of this section is to study the canonical cases described in Section 4 from the point of view of the AGM approach [25]. Afterwards, we focus on understanding the meaning of the various AGM postulates in terms of the changes we proposed. This will allow us to identify which of the AGM postulates are satisfied by our canonical cases, and which postulate instead have little meaning in our framework. (But, more in general, in a non-monotonic setting.)

This research issue is motivated, as introduced in Section 1, by the fact that the AGM postulates analysis in non-monotonic formalisms is still controversial, and thus open to discussion.

We recall that Delgrande proposed an approach to belief revision of logic programs under answer set semantics that is fully compliant with the base AGM postulates for revision [5]. He also claims in a later work [6, p. 568] that the third and fourth postulates 
for belief revision are not appropriate for belief revision of non-monotonic theories, and thus are ignored in his work. However, we are going to argue that these two postulates can be adopted in our approach, which suggests that the question whether the AGM postulates are suitable for non-monotonic reasoning is still open. Indeed, we shall prove that many other postulates are not satisfied. This is not surprising at all, given that they were thought with respect to a traditional logical setting; the meaning itself of such postulates is dubious when dealing with non-monotonic formalisations, but in general we shall show through our analysis that their meaning is not clear when trying to represent theory change in a non-monotonic setting.

However, we believe that it is important to describe the meaning of such postulates in terms of the logic/formalism under analysis to gain a better understanding of the nature of the various revision operations. Therefore, any result, positive or negative, about Defeasible Logic, constitutes an advancement of knowledge in this field, since the general agreement of scholars about inapplicability of AGM postulates to non-monotonic reasoning could be contradicted in that aspect.

In the remainder, we assume that the reader is familiar with the terminology used in the AGM framework, in particular with the notions of belief, belief set, and theory. (Note that in our framework the hypothesis of completeness of a theory does not hold in general, as it is perfectly normal that in a defeasible theory neither $+\partial p$ nor $+\partial \sim p$ are derivable.)

To adjust the AGM framework in the perspective of preference revision, we first rephrase the concept of extension into that of belief set corresponding to a defeasible theory.

Definition 24 Let $D=(F, R,>)$ be a defeasible theory. Then

$$
B S(D)=\left(B S^{+\partial}(D), B S^{-\partial}(D)\right)
$$

is the belief set of $D$, where

$$
\begin{aligned}
& B S^{+\partial}(D)=\{p \mid p \text { is a literal appearing in } D \text { and } D \vdash+\partial p\} \\
& B S^{-\partial}(D)=\{p \mid p \text { is a literal appearing in } D \text { and } D \vdash-\partial p\}
\end{aligned}
$$

We also state that when a literal $p$ is believed, $p \in K$ in AGM notation, then $p \in$ $B S^{+\partial}(D)$. Conversely, if a literal is not believed, i.e., $p \notin K$, then $p \in B S^{-\partial}(D)^{9}$. Intuitively, the idea is that if we prove $+\partial p$ then we believe in $p$, and if we prove $-\partial p$ then we do not believe in $p$.

An attentive reader will notice that this definition corresponds to the notion of extension of a theory $E(D)$. We hereafter shall use the notation of belief set $B S(D)$ to ease the parallel with the AGM paradigm. Moreover, we shall relate the AGM operators of contraction, expansion, and revision, and then reframe the corresponding postulates of AGM in the terminology of defeasible theories (in Sections 7.1-7.2).

\footnotetext{
${ }^{9}$ Note that it is possible that neither literal $p$ and $\sim p$ belong to $B S(D)$. For example, consider the theory consisting only of $p \Rightarrow p$ and $\neg p \Rightarrow \neg p$. In this theory none of $\pm \partial p$ and $\pm \partial \neg p$ is provable.
} 
Belief contraction is the process of rationally removing from a belief set $K$ a certain belief $\psi$ previously in the set. From the point of view of Defeasible Logic, by Definition 24 , a defeasible theory $D=(F, R,>)$ where $D \vdash+\partial p$ (i.e., $\left.p \in B S^{+\partial}(D)\right)$ is modified such that $-\partial p$ holds in the contracted theory (denoted by $D_{p}^{-}$) after the process (i.e., $p \in B S^{-\partial}\left(D_{p}^{-}\right)$). For the above reasoning, it seems reasonable to argue that the process of belief contraction as formalised in AGM approach corresponds to our first canonical case, i.e., from $+\partial p$ to $-\partial p$. If we consider a set of literals $C=\left\{p_{1}, \ldots, p_{n}\right\}$, we define the contracted theory $D_{C}^{-}$as the theory where for each $p_{i} \in C, p_{i} \in B S^{-\partial}\left(D_{C}^{-}\right)$.

Belief revision is the process of rationally deleting a certain belief $\psi$ from a belief set $K$ and adding its opposite. From the point of view of Defeasible Logic, by Definition 24 , a defeasible theory $D=(F, R,>)$ where $D \vdash+\partial \sim p$ (i.e., $\sim p \in B S^{+\partial}(D)$ and $p \in B S^{-\partial}(D)$ ) is modified such that $+\partial p$ holds in the revised theory (denoted by $D_{p}^{*}$ ) after the process (i.e., $p \in B S^{+\partial}\left(D_{p}^{*}\right)$ ). Remember that in Defeasible Logic $\sim p$ now belongs to $B S^{-\partial}\left(D_{p}^{*}\right)$. For the above reasoning, it seems reasonable to argue that the process of belief revision as formalised in AGM approach corresponds to our second canonical case, i.e., from $+\partial \sim p$ to $+\partial p$.

Belief expansion is the process of adding a certain belief $\psi$ to a belief set $K$. It is possible to consider two interpretations of the expansion process: the first where we simply force the belief in, the second where a belief is added if the opposite is not believed. Our third canonical case, i.e., from $-\partial p$ to $+\partial p$, follows the second strategy. Therefore, from the point of view of Defeasible Logic, by Definition 24 this process describes the case of an initial defeasible theory $D=(F, R,>)$ where $D \vdash-\partial p$ and $D \vdash-\partial \sim p$ (i.e., $p, \sim p \in B S^{-\partial}(D)$ ) hold being modified such that $+\partial p$ holds in the expanded theory (denoted by $D_{p}^{+}$) after the process (i.e., $p \in B S^{+\partial}\left(D_{p}^{+}\right)$). Remember that in Defeasible Logic $\sim p$ still belongs to $B S^{-\partial}\left(D_{p}^{+}\right)$. If we consider a set of literals $C=\left\{p_{1}, \ldots, p_{n}\right\}$, we define the expanded theory $D_{C}^{+}$as the theory where for each $p_{i} \in$ $C, p_{i} \in B S^{+\partial}\left(D_{C}^{+}\right)$.

\subsection{Preference Contraction}

Throughout this subsection, we assume that $D \vdash+\partial p$, for a literal $p$ in $D$.

The first postulate in AGM belief contraction states that when a belief set is contracted by a sentence $p$, the outcome should be logically closed. In Defeasible Logic, a theory $D$ is distinct from its extension $E(D)$ (i.e., its set of conclusions). In general, given an extension in Defeasible Logic, there are possibly different theories that could generate the same extension. This means that in AGM there is no difference between contracting a theory or its base, while this is not the case in Defeasible Logic. However, given that the allowed operations are either to add or to remove instances of the superiority relation, it is immediate to see that the contraction of a defeasible theory is a theory. The following version of the postulate holds for contraction of defeasible preferences.

$D_{p}^{-}$is a theory.

$(K \dot{-1})$

Notice that the definition of a defeasible theory does not impose any constraint on what properties the superiority relation must have. Hence, adding or removing tuples 
from a superiority relation results in another superiority relation and, consequently, the contracted theory is still a defeasible theory.

Note that contracting a literal does not necessarily result in removing instances from the superiority relation: sometimes you need to add tuples instead. Example 17 below shows one of such cases.

Example 17. Consider $D=(\emptyset, R, \emptyset)$, with $R=\{$

$$
\begin{aligned}
& \quad \Rightarrow_{r_{1}} p \\
& \Rightarrow_{r_{2}} \quad q \quad \Rightarrow_{r_{3}} \neg p \\
& \left.\Rightarrow_{r_{4}} \neg q\right\} .
\end{aligned}
$$

It is easy to verify that $D \vdash+\partial p$. Trivially, we cannot remove instances from an empty superiority relation. However, we can add the tuple $\left(r_{2}, r_{4}\right)$, and thus obtain $+\partial q$, which makes $r_{3}$ applicable. The new superiority relation still does not solve the conflict between $r_{1}$ and $r_{2}$ but now the theory so modified derives $-\partial p$.

Remark 1. As we pointed out in Proposition 1 and Example 3, sometimes the definition of defeasible theory requires the superiority relation to be acyclic. If one uses such a definition, then there are cases where contracting (and any other type of change in general) is only possible by introducing a cycle in the superiority relation, which, as we have seen in Proposition 1, could lead to an inconsistent theory. This means that the adoption of the postulate when there are restrictions on the superiority relation would exclude some otherwise possible operations (that could lead to possibly inconsistent theories).

Example 18. Let $D$ be a defeasible theory such that

$$
\begin{gathered}
\Rightarrow_{r_{1}} \quad p \\
a \Rightarrow_{r_{2}} \neg p .
\end{gathered}
$$

First, notice that $D \vdash-\varphi p$; thus for each chain for $p$, a rule supporting an opposite literal exists. (The example is clearly oversimplified by considering only one chain for $p$ and one chain attacking it, but it does not lose generality.) There is no assignment to the superiority relation able to contract $p$. This fact, again, lies in the difficulty of identifying "simple" schemas to capture the notion of tautological. Discussion hereinafter on Postulate $K \dot{-} 4$ will further investigate on this matter.

As preference contraction acts only on the superiority relation, to ensure that a contraction operation satisfies the postulate, we only have to check whether the operation itself does not create a cycle in the superiority relation. This is guaranteed by the following proposition.

Proposition 11 Given a defeasible theory $D=(F, R,>)$, if $D^{\prime}=\left(F, R,>^{\prime}\right)$ is obtained from $D$ by erasing preference tuples from $>$, then $>^{\prime}$ is acyclic.

Proof. By contradiction, let us suppose that there is a cycle in $>^{\prime}$. Since, by hypothesis, $>^{\prime}$ is obtained from $>$ by simply removing preference tuples, then each element of $>^{\prime}$ is an element of $>$ and the cycle in $>^{\prime}$ is also in $>$, against the hypothesis. 
The idea of the second AGM postulate for belief contraction is that, since a contraction removes beliefs, the contracted belief set is smaller than the original. AGM focuses only on "positive" beliefs. On the contrary, we showed that Defeasible Logics originates both a set of positive conclusions $E^{+}$as well as a set of negative ones $E^{-}$. We thus have to check for both of them.

$B S^{+\partial}\left(D_{p}^{-}\right) \subseteq B S^{+\partial}(D)$ and $B S^{-\partial}\left(D_{p}^{-}\right) \supseteq B S^{-\partial}(D)$.

This postulate does not hold: indeed, it contradicts the sceptical non-monotonic nature of Defeasible Logic, as shown in the example below.

Example 19. Consider $D=\left(\emptyset, R,>=\left(r_{2}, r_{1}\right),\left(r_{3}, r_{4}\right)\right)$, with $R=\{$

$$
\begin{array}{ccc}
\Rightarrow_{r_{1}} \neg a & \\
\Rightarrow_{r_{2}} a & \Rightarrow_{r_{3}} \quad p \\
& \vee \\
& \left.\Rightarrow r_{r_{4}} \neg p\right\} .
\end{array}
$$

Then $B S^{+\partial}=a, p$ and $B S^{+\partial}=\neg a, \neg p$. If we contract $a$ by erasing the superiority $r_{2}$ and $r_{1}$, then $\neg p$ becomes defeasibly provable, and we obtain $B S^{+\partial}=\neg p$ and $B S^{-\partial}(D)=a, \neg a, p .^{10}$

This behaviour is not confined to the specifics of Defeasible Logic, but holds, in general, for sceptical non-monotonic formalisms. When one contracts a literal, other literals depending on the contracted literal may be removed as well. If such literals were involved in conflicts, the opposite literals (1) are no longer opposed (attacked), and (2) can thus be included in the extension.

In classical-type of logics, notions of expanding or contracting a theory are symmetrically related with new pieces of information, you need to introduce new concepts within the theory itself. Symmetrically, contracting information always reflects

The third postulate of AGM considers the case when a belief $\psi$ is not in the initial belief set: if $\psi$ is not a consequence of the theory, then there is no reason to change anything at all. In Defeasible Logic, this corresponds to not being able to prove $p\left(p \in B S^{-\partial}(D)\right)$.

If $p \in B S^{-\partial}(D)$ then $B S\left(D_{p}^{-}\right)=B S(D)$.

$(K \dot{-} 3)$

The postulate trivially holds.

${ }^{10}$ There are (admittedly pathological) cases where, making a literal $p$ no longer defeasibly provable by changing the superiority relation, does not imply that $-\partial p$ holds after the revision process. For example, consider the theory $\Rightarrow_{r} p$ and $\neg p \Rightarrow_{s} \neg p$. The only way to prevent $+\partial p$ is to impose $s>r$, but in such a revised theory none of $+\partial p$ and $-\partial p$ would hold (same for $\neg p$ ). Notice that, in this case, the conditions for our canonical cases to succeed do not hold. 
The fourth AGM postulate states that the only literals that are immutable in the contraction process are tautologies. Defeasible Logic does not have logical connectives; therefore, it is not possible to have tautologies in the classical sense. Nevertheless, the concept of tautology is that of a statement that cannot ever be refuted: a tautology is true in every interpretation. In classical logic, an interpretation is an assignment of truth values to the propositional atoms, while in Defeasible Logic this corresponds to consider a particular set of propositional atoms as factual knowledge. In the context of this paper, where we assumed that the sets of facts and rules cannot be changed, the closest thing to an interpretation is an assignment of the superiority relation. Accordingly, a tautology in our setting is a literal that is defeasibly provable in every assignment of the superiority relation, or, in other terms, for every possible revision of the superiority relation. Formulating the postulate like "If $p$ is $>-R$-tautological, then $p$ holds in every revision", that would be trivially true. However, as Corollary 13 shows, the concept of being tautological cannot be represented by simple proof tags. Thus, we propose a formulation using our strongest proof tag, i.e. $+\Delta$. We give the formulation of the success postulate for contraction by using the contrapositive.

If $p \in B S^{+\partial}\left(D_{p}^{-}\right)$then $D \vdash+\Delta p$.

This postulate does not hold with that formulation: the concept of strict derivation embodied by $+\Delta$ does not fully capture the notion of tautology as a non-refutable statement. Indeed, there are cases where $D \vdash-\Delta p$ and $D \vdash+\partial p$, but we are still not able to obtain $D^{\prime}$ by only changing the superiority relation such that $D^{\prime} \vdash-\partial p$.

We then shift our attention on whether proof tag $+\varphi$ may fully interpret the concept of tautology in our framework; our choice is motivated by the fact that $+\varphi$ indeed denotes the presence of a supporting chain made of elements for which there are no rules for the opposite As such, it represents a non-refutable argument obtained from defeasible rules.

It thus seems reasonable to reformulate the success postulate for contraction as follows.

If $p \in B S^{+\partial}\left(D_{p}^{-}\right)$then $D \vdash+\varphi p . \quad\left(K \dot{-} 4^{\prime}\right)$

Even this version of the postulate does not cover all the cases in our framework. Indeed, there exist situations where for every proof of $p$ there exist a ( $\partial$-reachable) counter-argument, and yet it is not possible to change the theory in order to obtain $-\partial p$. A simple situation is to take a tautologous 3-SAT formula and to generate its $\Gamma$ transformation (see Definition 8). There are literals in the theory obtained that cannot be contracted. However, there are more cases.

Example 20. Consider $D=(\emptyset, R, \emptyset)$, with $R=\{$

$$
\begin{aligned}
\Rightarrow_{r_{1}} l & \Rightarrow r_{r_{2}} \neg a \\
& \Rightarrow r_{3} \quad a \Rightarrow r_{4} p \\
& \Rightarrow_{r_{5}} \quad b \quad \Rightarrow_{r_{6}} p \\
\Rightarrow_{r_{7}} \neg l & \left.\Rightarrow_{r_{8}} \neg b\right\} .
\end{aligned}
$$


It is trivial to see that $D \vdash-\varphi p$ due to $r_{2}$ and $r_{8}$. To contract $p$, we must block both the chains proving $p$. But, in order to do so, we should have that $D \vdash+\partial l$ as well as $D \vdash+\partial \neg l$. This is not possible since $D$ is consistent.

Unfortunately, the rules pattern shown in Example 20 is not a sufficient condition to reframe the postulate $\left(K \dot{-} 4^{\prime}\right)$. Indeed, as Example 21 shows, it is possible to find counter-examples where $p$ can be contracted, as well as counter-examples to counterexamples (we refer to Example 22) where, by extending the theory of Example 21 with rules $r_{19}, \ldots, r_{25}$, the contraction of $p$ becomes, again, not possible.

Example 21. Consider $D=(\emptyset, R, \emptyset)$, with $R=\{$

$$
\begin{aligned}
& \Rightarrow_{r_{1}} \quad a \quad \Rightarrow_{r_{2}} p \\
& \Rightarrow_{r_{3}} \quad b \quad \Rightarrow_{r_{4}} p \\
& \Rightarrow_{r_{5}} \quad c \quad \Rightarrow_{r_{6}} p \\
\Rightarrow_{r_{7}} \quad l & \Rightarrow_{r_{8}} \neg a \\
\Rightarrow_{r_{9}} \neg l & \Rightarrow_{r_{10}} \neg b \\
\Rightarrow_{r_{11}} \quad m & \Rightarrow_{r_{12}} \neg b \\
\Rightarrow_{r_{13}} \neg m & \Rightarrow_{r_{14}} \neg c \\
\Rightarrow_{r_{15}} n & \Rightarrow_{r_{16}} \neg c \\
\Rightarrow_{r_{17}} \neg n & \left.\Rightarrow_{r_{18}} \neg a\right\} .
\end{aligned}
$$

To contract $p$, we must block derivations of $+\partial a,+\partial b$ and $+\partial c$. This can be obtained by adding the following tuples to the superiority relation: $\left(r_{7}, r_{9}\right),\left(r_{11}, r_{13}\right)$ and $\left(r_{15}, r_{17}\right)$.

Example 22. Let $D^{\prime}$ be the theory of Example 21 where we add the following rules:

$$
\begin{aligned}
& \Rightarrow_{r_{19}} \quad e \Rightarrow_{r_{20}} p \\
& { }_{r_{21}} f \Rightarrow_{r_{22}} p \\
n & \Rightarrow_{r_{23}} \neg e \\
\neg n & \Rightarrow_{r_{24}} \neg f \\
\neg m & { }_{r_{25}} \neg f .
\end{aligned}
$$

To contract $p$, we must now block derivations also of $+\partial e$, and $+\partial f$. The proof of $e$ can be blocked only if we prove, $n$ the antecedent of $r_{23}$. (In this way, the derivation of $c$ would be blocked as well.) This implies that the only way to block the derivation of $f$ is by proving $\neg m$ (the only antecedent of rule $r_{25}$ ). We can now operate only on the provability of either $l$, or $\neg l$. In both cases, one between $a$ or $b$ cannot be refuted.

This is in line with Corollary 13, which thus implies that there is not a simple condition, based on proof tags, that can be computed in polynomial time and also guarantees a successful contraction.

The fifth AGM postulate states that contracting, and then expanding by the same belief $\psi$ will give back at least the initial theory.

If $p \in B S^{+\partial}(D)$ then $B S(D) \subseteq B S\left(\left(D_{p}^{-}\right)_{p}^{+}\right)$.

$(K \dot{-} 5)$ 
This postulate cannot be adopted since, once the contracted theory has been obtained, the backward step does not uniquely correspond to expanding the obtained theory by the same literal, as the following example shows.

Example 23. Consider $D=\left(\emptyset, R,\left\{\left(r_{1}, r_{3}\right\}\right)\right.$, with $R=\{$

$$
\begin{aligned}
& \Rightarrow_{r_{1}} \quad a \quad \Rightarrow_{r_{2}} p \\
& \vee \\
& \Rightarrow_{r_{3}} \neg a \\
& \Rightarrow_{r_{4}} \quad b \quad \Rightarrow_{r_{5}} p \\
& \left.\Rightarrow_{r_{6}} \neg b\right\} .
\end{aligned}
$$

If we contract $D$ by $p$, one possible solution is to erase tuple $\left(r_{1}, r_{3}\right)$ from $>$. If we now expand $D_{p}^{-}$, one solution is indeed the initial theory, but another solution would be the theory such that $>=\left\{\left(r_{4}, r_{6}\right)\right\}$.

Nevertheless, if all operations in the contraction process can be traced, then we can easily backtrack and obtain the initial theory, satisfying the postulate.

The sixth AGM postulate, also known as the postulate of the irrelevancy of syntax, states that if two beliefs $\psi$ and $\chi$ are logically equivalent, then contracting by $\psi$ and contracting by $\chi$ produce the same result.

If $\vdash p \equiv q$ then $B S\left(D_{p}^{-}\right)=B S\left(D_{q}^{-}\right)$.

In Defeasible Logic, the language is restricted to literals, thus two elements $p$ and $q$ are equivalent only if they represent the same literal. For this reason, the sixth postulate trivially follows.

The seventh and the eighth postulate are best understood if seen in combination. They essentially relate two individual contractions with respect to a pair of sentences $\psi$ and $\chi$, with the contraction of their conjunction $\psi \wedge \chi$. As already stated, in Defeasible Logic there are no logical connectives and a conjunction of literals is equivalent to the set of the same literals; the same reasoning used to introduce postulate $(K \dot{-} 2)$ applies here. Thus, the two postulates can be rewritten as

$B S^{+\partial}\left(D_{p}^{-}\right) \cap B S^{+\partial}\left(D_{q}^{-}\right) \subseteq B S^{+\partial}\left(D_{p, q}^{-}\right)$and
$B S^{-\partial}\left(D_{p}^{-}\right) \cap B S^{-\partial}\left(D_{q}^{-}\right) \supseteq B S^{-\partial}\left(D_{p, q}^{-}\right)$

If $p \in B S^{-\partial}\left(D_{p, q}^{-}\right)$then $B S^{+\partial}\left(D_{p, q}^{-}\right) \subseteq B S^{+\partial}\left(D_{p}^{-}\right)$and $B S^{-\partial}\left(D_{p}^{-}\right) \subseteq B S^{-\partial}\left(D_{p, q}^{-}\right)$.

Postulates $(K \dot{-} 7)$ and $(K \dot{-} 8)$ do not hold for the same reason of postulate $(K \dot{-} 2)$. The following example shows the truth this for both of them.

Example 24. Consider $D=\left(\emptyset, R,\left\{\left(r_{1}, r_{0}\right),\left(r_{2}, r_{3}\right),\left(r_{4}, r_{6}\right),\left(r_{8}, r_{5}\right),\left(r_{7}, r_{9}\right)\right\}\right)$, with $R=$ \{ 


$$
\begin{array}{ccc}
\Rightarrow_{r_{0}} & \neg a & \\
\wedge & & \\
\Rightarrow_{r_{1}} \quad a & \Rightarrow_{r_{2}} \quad c \\
& \vee \\
& \Rightarrow_{r_{3}} \neg c \\
& \Rightarrow_{r_{5}} \neg d \\
& \wedge \\
\Rightarrow_{r_{7}} \quad b \quad{ }_{r_{8}} d \\
\left.\vee_{r_{9}} \neg b\right\} .
\end{array}
$$

We have $B S^{+\partial}(D)=\{a, b, c, d, \neg p\}$ and $B S^{-\partial}(D)=\{\neg a, \neg b, \neg c, \neg d, p\}$. Let us contract $D$ by literal $a$ and by literal $b$ (where the contractions are minimal with respect to the changes in the superiority relation) obtaining:

$$
\begin{aligned}
& B S^{+\partial}\left(D_{a}^{-}\right)=\{b, \neg c, d, \neg p\} \\
& B S^{+\partial}\left(D_{b}^{-}\right)=\{a, c, \neg d, \neg p\} \\
& B S^{-\partial}\left(D_{a}^{-}\right)=\{a, \neg a, \neg b, c, \neg d, p\} \\
& B S^{-\partial}\left(D_{b}^{-}\right)=\{\neg a, b, \neg b, \neg c, d, p\} .
\end{aligned}
$$

The respective intersections are:

$$
\begin{aligned}
& B S^{+\partial}\left(D_{a}^{-}\right) \cap B S^{+\partial}\left(D_{b}^{-}\right)=\{\neg p\} \\
& B S^{-\partial}\left(D_{a}^{-}\right) \cap B S^{-\partial}\left(D_{b}^{-}\right)=\{\neg a, \neg b, p\} .
\end{aligned}
$$

We can now contract $a$ and $b$ simultaneously, and obtain

$$
\begin{aligned}
& B S^{+\partial}\left(D_{a, b}^{-}\right)=\{\neg c, \neg d, p\} \\
& B S^{-\partial}\left(D_{a, b}^{-}\right)=\{a, \neg a, b, \neg b, c, d, \neg p\}
\end{aligned}
$$

proving our claim.

Throughout postulates $(K \dot{-} 1)$ to $(K \dot{-} 8)$ we took care of the effects of the contraction process, due to the specific nature of positive and negative beliefs in Defeasible Logic. For each postulate, this specificity has however no effect. In fact, what can be claimed for contractions in $B S^{+\partial}$ extends to $B S^{-\partial}$, and the other way around.

For the sake of completeness, we apply the same care to expansion and revision cases further on. As it will be clear at the end of each analysis, analogous conclusions about the redundancy are derived.

\subsection{Preference Revision}

Throughout this subsection, we assume that $D \vdash+\partial \sim p$ and $D \vdash+\Sigma p$ for a $\partial$-reachable literal $p$ in $D$. (The last two assumptions restrict the scope of investigation to literals for which there exist (at least) a contradiction-free supporting chain.) 
The first AGM postulate for revision states that the revision process must preserve the logical closure of the initial theory.

$D_{p}^{*}$ is a theory.

We can repeat the argument (and the related remark) for postulate $K \dot{-1}$ for the justification of the reasons why this postulate holds.

The second AGM postulate for revision captures the most general interpretation of theory change; the new information $\psi$ is always included in the new belief set, even if $\psi$ is self-inconsistent, or contradicts some belief of the initial theory. As a consequence, the complete reliability of the new information is always assumed.

$p \in B S^{+\partial}\left(D_{p}^{*}\right)$.

As by definition of our second canonical case, literal $p$ is forced to be defeasibly proved after the process, provided that preconditions $+\partial \sim p$ and $+\Sigma p$ hold $p$ is $\partial$ reachable, the postulate is clearly satisfied.

The third and the fourth postulates of AGM revision explain the relationship between the revision and the expansion processes.

$B S^{+\partial}\left(D_{p}^{*}\right) \subseteq B S^{+\partial}\left(D_{p}^{+}\right) . \quad(K * 3)$

If $\sim p \in B S^{-\partial}(D)$ then $B S^{+\partial}\left(D_{p}^{+}\right) \subseteq B S^{+\partial}\left(D_{p}^{*}\right) . \quad(K * 4)$

Both the first two canonical cases, starting from an initial theory and considering a literal $p$, operate to obtain a final theory where $+\partial p$ holds. What we have to care about, however, are the preconditions under which these two canonical cases apply. The third postulate essentially states that every belief that can be derived revising a theory by a belief $\psi$ can also be obtained by expanding the same initial theory with respect to the same belief. This statement is perfectly allowed in our framework; the case where both revision and expansion can apply is when $+\partial \sim p$ (and hence $-\partial p$ ) holds in the initial theory. In this case, the two processes behave in the same manner, i.e., they calculates the same extensions. If we nonetheless regard at proper expansion, i.e., when condition $-\partial \sim p$ holds, then it is easy to see that the preconditions for expansion and revision are mutually exclusive: they cannot be applied at the same time.

The fifth AGM postulate states that the result of a revision by a belief $\psi$ is the absurd belief set if the new information is in itself inconsistent.

If $p$ is consistent then $B S^{+\partial}\left(D_{p}^{*}\right)$ is also consistent.

The first issue to address here is what it means for a literal to be consistent. We consider two alternatives: (i) the classical reading, where a literal is always consistent, (ii) a literal $p$ is consistent in a theory if the theory does not prove both $+\partial p$ and $+\partial \neg p$. However, since Defeasible Logic is para-consistent (see Example 3) this postulate holds for none of the alternatives. Example 25 provides a counter-example. 
Example 25. Consider $D=\left(\emptyset, R,\left\{\left(r_{3}, r_{1}\right)\right\}\right)$, with $R=\{$

$$
\begin{aligned}
& \begin{array}{ccc} 
& & \Rightarrow_{r_{1}} \neg p \\
\Rightarrow_{r_{2}} & a \wedge
\end{array} \\
& a, q, \neg q \Rightarrow_{r_{3}} p \\
& \Rightarrow r_{4} \quad q \\
& \left.\Rightarrow_{r_{5}} \neg q\right\} \text {. }
\end{aligned}
$$

We have $+\partial \neg p$ and $-\partial p$. The only way to conclude $+\partial p$ is to add $\left(r_{4}, r_{5}\right)$ and $\left(r_{5}, r_{4}\right)$ to $>$. Then, in the revised theory we conclude $+\partial p,-\partial \neg p,+\partial q$ and $+\partial \neg q$. Hence, the theory is inconsistent, but $p$ is consistent with the theory.

On the other hand, if one adopt the condition of Remark ??, then the postulate is trivially satisfied, since when we operate on a theory, the resulting object, when the superiority relation is cyclic what we could obtain might not be a theory (the revision operator generates a cycle in the transitive closure of the superiority relation).

The sixth AGM postulate for revision follows the same idea of $(K \dot{-} 6)$ : The syntax of the new information has no effect on the revision process, all that matters is its content. Again, the postulate has a natural counterpart in Defeasible Logic.

If $\vdash p \equiv q$ then $B S^{+\partial}\left(D_{p}^{*}\right)=B S^{+\partial}\left(D_{q}^{*}\right)$.

The reasoning is the same exploited in the counterpart postulate for contraction, and the postulate trivially holds.

The seventh and the eight postulate of AGM revision cope with the revision process with respect to conjunctions of literals. In the classical AGM framework, the principle of minimal change takes an important role in the formulation of these postulates. The revision with both $\psi$ and $\chi$ should correspond to a revision of the theory with $\psi$ followed by an expansion by $\chi$, provided that $\chi$ does not contradict the beliefs in the theory revised by $\psi$.

$B S^{+\partial}\left(D_{p, q}^{*}\right) \subseteq B S^{+\partial}\left(\left(D_{p}^{*}\right)_{q}^{+}\right)$and $B S^{-\partial}\left(\left(D_{p}^{*}\right)_{q}^{+}\right) \subseteq B S^{-\partial}\left(D_{p, q}^{*}\right)$.

If $\neg q \in B S^{-\partial}\left(D_{p}^{*}\right)$ then $B S^{+\partial}\left(\left(D_{p}^{*}\right)_{q}^{+}\right) \subseteq B S^{+\partial}\left(D_{p, q}^{*}\right)$ and $B S^{-\partial}\left(D_{p, q}^{*}\right) \subseteq B S^{-\partial}\left(\left(D_{p}^{*}\right)_{q}^{+}\right)$.

Again, these postulates cannot be satisfied. The following example gives a specific case that falsifies them. 
Example 26. Consider $D=(\emptyset, R, \emptyset)$ with $R=\{$

$$
\begin{aligned}
& \Rightarrow_{r_{1}} \neg a \\
& \Rightarrow_{r_{2}} \quad a \Rightarrow_{r_{3}} \quad p \\
& \Rightarrow r_{4} \neg p \\
& \Rightarrow_{r_{5}} \quad b \\
& \Rightarrow r_{6} \neg b \\
& b \Rightarrow_{r_{7}} p \\
& b \Rightarrow_{r_{8}} q \\
& \Rightarrow_{r_{9}} \neg q \\
& \left.\Rightarrow_{r_{10}} c \Rightarrow_{r_{11}} q\right\} \text {. }
\end{aligned}
$$

We have $B S^{+\partial}(D)=\{\neg p\}$, while all other literals belong to $B S^{-\partial}(D)$. If $D$ needs to be revised for $p$ and $q$, a possible theory is $D_{p, q}^{*}$, obtained by operating through the provability of literal $b$ and by updating the superiority relation to $>_{p, q}^{*}=\left\{\left(r_{5}, r_{6}\right),\left(r_{7}, r_{4}\right),\left(r_{8}, r_{9}\right)\right\}$. The resulting $B S^{+\partial}\left(D_{p, q}^{*}\right)=\{b, c, p, q\}$, while $B S^{-\partial}\left(D_{p, q}^{*}\right)=\{a, \neg a, \neg b, \neg p, \neg q\}$.

Let us now consider the revision only by $p$. A possible solution is $D_{p}^{*}$ such that $B S^{+\partial}\left(D_{p}^{*}\right)=\{a, c, p\}$ and $B S^{-\partial}\left(D_{p}^{*}\right)=\{\neg a, b, \neg b, \neg p, q, \neg q\}$. In this case, the revision process acts on the provability of literal $a$, by updating the superiority relation to $>_{p}^{*}=$ $\left\{\left(r_{2}, r_{1}\right),\left(r_{3}, r_{4}\right)\right\}$.

If we now expand $D_{p}^{*}$ by $q$, a possible solution is to add $\left(r_{11}, r_{9}\right)$ to $>_{p}^{*}$. By doing so we would obtain $B S^{+\partial}\left(\left(D_{p}^{*}\right)_{q}^{+}\right)=\{a, c, p, q\}$ and $B S^{-\partial}\left(\left(D_{p}^{*}\right)_{q}^{+}\right)=\{\neg a, b, \neg b, \neg p, \neg q\}$. The intersection of $D_{p, q}^{*}$ and $\left(D_{p}^{*}\right)_{q}^{+}$is not empty, but neither theory is contained in the other.

\subsection{Preference Expansion}

Throughout this subsection, we assume that for a $\partial$-reachable literal $p$ in $D: D \vdash-\partial p$, $D \vdash-\partial \sim p$ and $D \vdash+\Sigma p$.

The first AGM postulate for expansion states that if a theory is expanded with a belief $\psi$, then the resulting theory is the logical closure of the initial theory.

$D_{p}^{+}$is a theory.

$$
(K+1)
$$

Again, the remarks and reasons for Postulate $K \dot{-} 1$ apply for this postulate as well.

The second AGM postulate for expansion ensures that the "expansion" belief $\psi$ always belongs to the belief set of the resulting theory.

$p \in B S^{+\partial}\left(D_{p}^{+}\right)$.

$(K+2)$

Due to the hypotheses of $D \vdash+\Sigma p$ and $p$ being $\partial$-reachable, the postulate trivially holds since the expansion process forces literal $p$ to be defeasibly proved. 
The joint formulation of the third and the fourth AGM postulates for expansion states that if a belief is already present in the initial belief set, then the theory remains unchanged after the expansion process.

$B S^{+\partial}(T) \subseteq B S^{+\partial}\left(T_{p}^{+}\right)$and $B S^{-\partial}\left(T_{p}^{+}\right) \subseteq B S^{-\partial}(T) . \quad(K+3)$

If $p \in B S^{+\partial}(T)$ then $B S^{+\partial}\left(T_{p}^{+}\right) \subseteq B S^{+\partial}(T)$ and $B S^{-\partial}(T) \subseteq B S^{-\partial}\left(T_{p}^{+}\right) . \quad(K+4)$

Since we aim at obtaining a theory where $+\partial p$ holds, and by hypothesis $p \in$ $B S^{+\partial}(T)$, the postulates seen together trivially hold given that, by definition, their premises do not.

The fifth AGM postulate states that if a belief set is contained in another one, then the expansion of both sets wrt. the same belief preserves the inclusion relation.

If $B S^{+\partial}(D) \subseteq B S^{+\partial}\left(D^{\prime}\right)$ then $B S^{+\partial}\left(D_{p}^{+}\right) \subseteq B S^{+\partial}\left(D_{p}^{\prime+}\right) . \quad(K+5)$

Again, due the non-monotonic nature of Defeasible Logic, this postulate can not be satisfied, as already pointed out for Postulate $(K \dot{-} 2)$.

The sixth AGM postulate assures the minimality of the expanded belief set. Defeasible Logic derives conclusions that are tagged. The specific nature of this tagging is that it makes the notion of minimality for a set of conclusions useless. We can consider minimality only for one given tag, and not for all tags. The idea of "smallest resulting set" is consequently meaningless for non-monotonic systems tagging conclusions.

Given a theory $D$ and a belief $p$, $B S\left(D_{p}^{+}\right)$is the smallest belief set satisfying $(K+1)-(K+5)$.

$(K+6)$

In the perspective of non-monotonic reasoning, the operation of expanding a defeasible theory in order to prove a literal $p$ necessarily falsifies some other literals, previously provable in the initial theory; hence, the postulate cannot hold in general.

We end this part by presenting Table 2 where we summarise the results obtained so far concerning which postulates hold and which do not. (In the latter case, the last column reports the specific examples where counter-examples can be found.)

It is important to notice that all the examples proposed can be easily accommodated to show a more general result: the AGM postulates do not hold in general for a nonmonotonic setting, even when other operations rather than changing only the superiority relation are allowed. For instance, let us reformulate the theory of Example 19 while considering Postulate $K \dot{-} 2$.

$$
\begin{array}{cc}
\Rightarrow_{r_{1}} \neg a & \\
\wedge_{r_{2}} a & \Rightarrow_{r_{3}} \quad p \\
& \vee \\
& \Rightarrow r_{r_{4}} \neg p \\
b \Rightarrow_{r_{5}} \neg a . &
\end{array}
$$




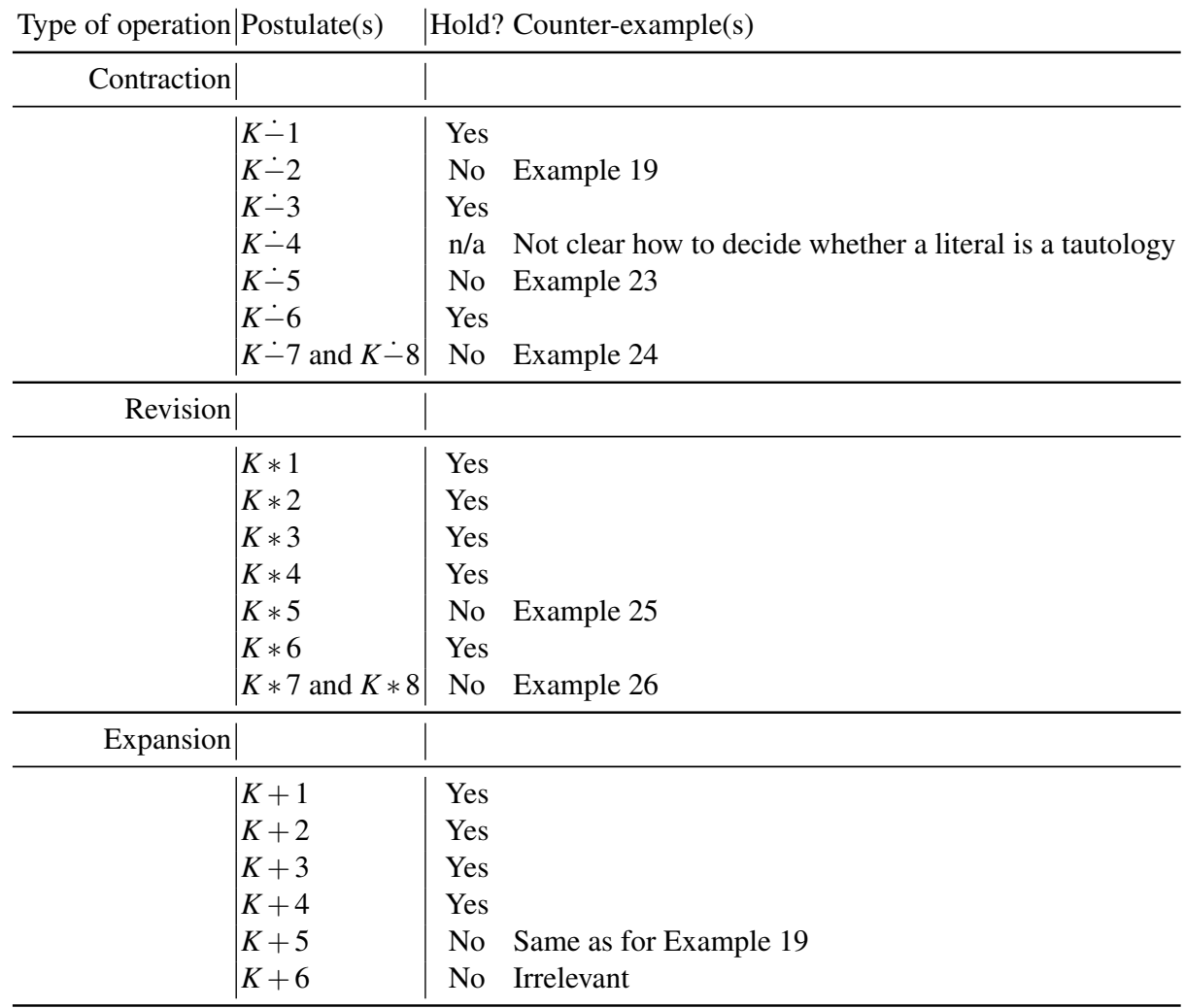

Table 2: Summary of AGM postulates for preference revision

Instead of erasing $r_{2}>r_{1}$ for the superiority relation, we can add $b$ to the set of facts, allowing $r_{5}$ to be applicable. Even in this case, Postulate $K \dot{-} 2$ does not hold.

\subsection{Identities for preference change}

In the AGM framework it is possible to define some change operation in terms of the others. The aim of this section is to investigate to what extent this is possible in the framework we proposed in this paper.

In the AGM framework a process that defines revision in terms of expansion is available, suggested by Isaac Levi in [26]. The idea being that, in order to revise a theory $D$ by a belief $\psi$, we may firstly contract $D$ by $\neg \psi$ in order to remove any information that may contradict $\psi$, and then expand the resulting theory with $\psi$. This procedure is known as the Levi identity which can be reformulated in out terminology as follows:

$B S\left(D_{p}^{*}\right)=B S\left(\left(D_{\neg p}^{-}\right)_{p}^{+}\right)$.

The following example shows that the Levi identity does not hold in our framework. 
Example 27. Consider $D=(\emptyset, R, \emptyset)$, with $R=\{$

$$
\begin{aligned}
& \Rightarrow_{r_{1}} \quad a \quad \Rightarrow_{r_{2}} \quad p \\
& \Rightarrow_{r_{3}} \neg a \\
& \quad \Rightarrow_{r_{4}} \neg p \\
& \Rightarrow_{r_{5}} \quad b \quad \Rightarrow_{r_{6}} p \\
& \left.\Rightarrow_{r_{7}} \neg b\right\} .
\end{aligned}
$$

Here, $D \vdash-\partial p$ and $D \vdash+\partial \neg p$. If we revise $D$ by $p$, a possible solution is $D_{p}^{*}$ such that $B S^{+\partial}\left(D_{p}^{*}\right)=\{a, p\}$, and $B S^{-\partial}\left(D_{p}^{*}\right)=\{\neg a, b, \neg b, \neg p\}$. Now, contracting $D$ by $\neg p$ can lead to $D_{\neg p}^{-}$with $B S^{+\partial}\left(D_{\neg p}^{-}\right)=\{b\}$, and $B S^{-\partial}\left(D_{\neg p}^{-}\right)=\{a, \neg a, \neg b, p, \neg p\}$. If we expand $D_{\neg p}^{-}$by $p$, we obtain $\left(D_{\neg p}^{-}\right)_{p}^{+}$with $B S^{+\partial}\left(\left(D_{\neg p}^{-}\right)_{p}^{+}\right)=\{b, p\}$, and $B S^{-\partial}\left(\left(D_{\neg p}^{-}\right)_{p}^{+}\right)=$ $\{a, \neg a, \neg b, \neg p\}$.

The Levi identity does not hold as our revision procedure concerns the reasons why one belief is obtained, and not only whether we have that one belief. Accordingly, if there are multiple reasons to justify one belief, it is possible to contract the theory in multiple ways and similarly to expand it in multiple ways. In a non-monotonic setting, the changes for the contractions are not necessarily the "opposite" of those for contraction.

As Levi Identity relates the revision process in terms of expansion, Harper proposed a method to obtain the contraction by using revision [27]; the underlying idea is that a theory $D$ contracted by a belief $\psi$ is equivalent to the theory containing only the information that remain unchanged during the process of revising $D$ by $\neg \psi$. In our terms, the Harper Identity can be rewritten as

$B S\left(D_{p}^{-}\right)=B S\left(D_{\neg p}^{*}\right) \cap B S(D)$.

Even Harper Identity does not hold for the operations defined in this paper, and Example 28 provides a counter-example to Harper Identity.

Example 28. Consider $D=\left(\emptyset, R,\left\{\left(r_{1}, r_{4}\right),\left(2_{2}, r_{3}\right),\left(r_{5}, r_{3}\right)\right\}\right.$, with $R=\{$

$$
\begin{aligned}
& \Rightarrow_{r_{1}} p \Rightarrow_{r_{2}} q \\
& \vee \quad \Rightarrow_{r_{3}} \neg q \\
& \left.\Rightarrow_{r_{4}} \neg p \Rightarrow_{r_{5}} q\right\} \text {. }
\end{aligned}
$$

The initial belief set is $B S^{+\partial}(D)=\{p, q\}$ and $B S^{-\partial}(D)=\{\neg p, \neg q\}$. If we contract $D$ by $p$, we obtain a theory $D_{p}^{-}$such that $B S^{+\partial}\left(D_{p}^{-}\right)$is $\{\neg q\}$ and $B S^{-\partial}\left(D_{p}^{-}\right)$contains all the other literals. Instead, if we revise the initial theory with $\neg p$ the theory $D_{\neg p}^{*}$ where $B S^{+\partial}\left(D_{\neg p}^{*}\right)=\{\neg p, q\}$ and $B S^{-\partial}\left(D_{\neg p}^{*}\right)=\{p, \neg q\}$ is obtained. The intersections between the revised theory and the initial one are $\left.B S^{+\partial} D_{\neg p}^{*}\right) \cap B S^{+\partial}(D)=\{q\}$ and $B S^{-\partial}\left(D_{\neg p}^{*}\right) \cap B S^{-\partial}(D)=\{\neg q\}$. 
Again, the main reason for the failure of the Harper Identity lies in the non-monotonic nature of Defeasible Logic where, in general, it is not possible to control the consequences of a given formula.

In this section, we provided an interpretation of the AGM postulates for expansion, contraction and revision in terms of our canonical cases and the operations that are possible when the changes operate only on the superiority relation.

We believe that the contribution of this section is multi-fold. First of all, the definition of our canonical cases offers a more precise formal understanding of the intuition of the various operations. Second, we reconstructed the postulates for the canonical cases, and discussed how to adapt them.

Note that while the main analysis in this paper is specific to revision of the superiority relation of Defeasible Logic, the definition of the canonical cases does not depend on it, and it can be applied in a much broader context. For instance, the canonical case from $+\partial p$ to $+\partial \neg p$ can be understood as "how do we modify a theory such that before the revision a formula holds, and after the revision the opposite holds?"; similarly for the other canonical cases.

The last contribution of the analysis confirms the outcome of [3], showing that, typically, the postulates describing inclusion relationships between belief sets before and after a revision operation do not hold for Defeasible Logic, and it is unreasonable to expect that they hold for non-monotonic reasoning in general.

\section{Related Work}

As far as we are aware of, the work most closely related to ours is that of [28] where the authors study how to abduct a preference relation to support the derivation of a specific conclusion. The problem they address is however conceptually different from what we presented in this paper, given that we focused on the modification of the superiority relation. The mentioned investigation provides a framework where a preference that is abduced applies to the inferential process, whilst we provide a method to modify a preference in order to obtain a specific inference. Note that in non-monotonic reasoning, a revision is not necessarily triggered by inconsistencies. [3] investigates revision for Defeasible Logic and relationships with AGM postulates. While their ultimate aim is similar to that of the present paper - i.e., transforming a theory to make a previously provable (resp. non provable) statement, non provable (resp. provable) - the approach is different, and more akin to standard belief revision. More precisely, revision is achieved by introducing new exceptional rules. Furthermore, they discuss how to adapt the AGM postulates for non-monotonic reasoning.

Our work is motivated by legal reasoning, where preference revision is just one of the aspects of legal interpretation. [29,30] propose a Defeasible Logic framework to model extensive and restrictive legal interpretation. This is achieved by using revision mechanisms on constitutive rules, where the mechanism is defined to change the strength of existing constitutive rules. Based on the specific type of norm to modify, they propose a revision (contraction) operator which modifies the theory by adding (removing) facts, strict rules, or defeaters, raising the question whether extensive and 
restrictive interpretation can be modelled as preference revision operators. An important aspect of legal interpretation is finding the legal rules to be applied in a case. (In this work we assumed that the relevant rules have already been discovered, and in case of conflicts, preference revision can be used to solve them.)

Another work, related to revision of Defeasible Logic is that of [4], where the key idea is to model revision operators corresponding to typical changes in the legal domain, specifically, abrogation and annulment. The authors show that, typically, belief revision methodologies are not suitable to changes in theories intended for legal reasoning. They show that it is possible to revise theories fully satisfying the AGM postulates, but then the outcome is totally meaningless from a legal point of view.

The connection between sceptical, non-monotonic formalisms and argumentation is well known in literature; in [12], the authors adapt Dung's argumentation framework [31,32] to give an argumentation semantics for Defeasible Logic: first, they prove that Dung's grounded semantics characterises the ambiguity propagating $D L$; then, they show that the ambiguity blocking $D L$ is described with an alternative notion of Dung's acceptability. The main effort was to establish close connections between defeasible reasoning and other formulations of non-monotonic reasoning.

Non-monotonic revision through argumentation was also investigated in [9] by using Defeasible Logic Programming (DELP). They define an argument revision operator that inserts a new argument into a defeasible logic program in such a way that such an argument ends up undefeated after the revision, thus warranting its conclusion. (A conclusion $\alpha$ is warranted if there exists a non-defeated argument supporting it.) Their concept of defeaters denotes stronger counter-arguments to a given conclusion based on a set of preferences stating which argument prevails against one other. Their work suffers from a main drawback: imposing preferences among arguments (i.e., whole reasoning chains in our framework), instead of single rules, can lead to a situation when an argument is warranted even if all its sub-arguments are defeated. The DELP formalism is very similar to Defeasible Logic. Techniques proposed in our work can thus be easily accommodated to join the framework presented in $[33,34]$.

Other works closely related to ours are [35,36,37,38]. They propose extensions of an argumentation framework, Defeasible Logic and Logic Programming, where the superiority relation is dynamically derived from arguments and rules in given theories. While the details are different for the various approaches, the underlying idea is the same. For example, in [36], it is possible to have rules of the form $r: a \Rightarrow(s>t)$ where $s$ and $t$ are identifiers for rules. Accordingly, to assert that rule $s$ is stronger than rule $t$ we have to be able to prove $+\partial a$ and that there are no applicable rules for $\neg(s>t)$. In addition, the inference rules require that instances of the superiority relation are provable (e.g., $+\partial(s>t)$ ) instead of being simply given (as facts) in $>$, that is $(s, t) \in>$. The main difference with these works is that we investigate general conditions under which it is possible to modify the superiority relation in order to change the conclusions of a theory, while they provide specific mechanisms to compute conclusions where the preference relations are inferred from the context. They do not study which the possible ways to revise a theory are. For instance, if a literal is $>$-tautological, no matter how we derive instances of the preference relation, there is no way to prevent its derivation, or to derive its negation. 
In the scenario where the preferences over rules are computed dynamically, one could argue that it might be possible to encode in the theory the possible ways in which the superiority relation would behave. The problem with this approach is the combinatorial explosion of the number of rules required, since one would have to consider rules with the form $a_{1}, \ldots, a_{n} \Rightarrow\left(r_{i}>r_{j}\right)$ for all possible combinations of literals $a_{k}$ in the theory, and also for all possible combinations of instances of $>$. In both cases there is an exponential number of combinations. Among the works mentioned above, [35] is motivated, as are we, by legal reasoning, and they use rules to encode the legal principles we shortly discussed in the introduction.

\section{Conclusions and further work}

Over the years Defeasible Logic has proved to be a simple but effective practical nonmonotonic formalism suitable for applications in many areas. Since its first formulation in [39], many theoretical aspects of Defeasible Logic have been studied: from its proof theory [16] to relationships to logic programming [24], from variants of the logic [21] to its semantics [12] and computational properties [23]. Furthermore, several efficient implementations have been developed [40,41,42]. Methods to revise, contract, or expand a defeasible theory were first proposed in [3], where the authors studied how to revise the belief set of a theory based on introduction of new rules. The resulting methodology was then compared to the AGM belief revision framework.

In the present work, we took a different approach: since, in many situations, a person cannot change the rules governing a system (a theory) but only the way each rule interact with the others, it seems straightforward to consider revision methodologies of Defeasible Logic where derivation rules are considered as "static" or "untouchable", and the only way to change a theory with respect to a statement is to modify the relative strength of a rule with respect to another rule, that is how to modify the superiority relation of the analysed theory.

We therefore presented in Section 3 the formalism adopted: Eight different types of tagged literals were described to simplify the categorisation process and, consequently, the revision calculus. In Section 4, we introduced three canonical cases of possible revisions and systematically analysed every canonical instance. In both sections, we presented several theoretical results on conditions under which a revision process is possible. One of the main result consisted in proving that the problem of revising a defeasible theory by only changing the superiority relation is NP-complete. This is in line with the struggle with the general problem of strategic argumentation. In the setting of two discussants arguing about the truthfulness of a claim, [11,43] proved that the problem of deciding which set of arguments to play at each turn is NP-complete. ([44] went further by proving that the problem remains NP-complete even in case of espionage or collusion.)

Upon these theoretical basis, in Section 7, we proposed a systematic comparison between our framework and the AGM postulates. In there, the three canonical cases were compared to the AGM contraction, expansion, and revision operators: For each belief change operator, all the AGM postulates were rewritten using our terminology, and their validity was studied in our framework. 
The work presented in this paper paves the way to several lines of further investigation to extend the proposed change methodologies.

The first extension we want to mention regards changing the status of a sets of literals instead of a single literal. Studying conditions (supporting chains, proof tags, and so on) to understand when, and where, it is possible to change a theory by more than a single literal is not a trivial issue.

Example 29. Consider $D=\left(\emptyset, R,\left\{\left(r_{6}, r_{3}\right)\right\}\right)$, with $R=\{$

$$
\begin{aligned}
& \quad \Rightarrow_{r_{1}} \neg b \Rightarrow_{r_{2}} q \\
& \Rightarrow_{r_{3}} \quad a \quad r_{r_{4}} \quad b \Rightarrow_{r_{5}} p \\
& \left.\wedge_{r_{6}} \neg a\right\} .
\end{aligned}
$$

Since $p$ depends on $b$, and symmetrically $q$ depends on $\neg q$, it is not possible to change the initial theory if we want to obtain both $+\partial p$ and $+\partial q$.

The second extension concerns how to limit the scope of the revision operators. Revision of preferences should not involve minimal defeasible rules. This constraint captures the idea that a rule that wins against all other rules is a basic juridical principle. A similar aspect is that, under given circumstances, the revision process should not, for at least a subset of "protected" pairs, violate the original preferential order. For instance, we should not revise those preferences that are unquestioned because derived by commonly accepted principles or explicitly expressed by the legislator, as discussed in the introduction.

As we have seen in Section 2, in the legal domain we can identify several sources for the preference relation. Preference handling in Defeasible Logic can gain much from typing of preferences themselves. The notion of preference type and its algebraic structure has been studied previously and can be applied directly here [45]. Analogously, one of the possible directions of generalisation for the notion of preference is the notion of partial order, investigated at a combinatorial level by [46] and then studied from a computational viewpoint in [47].

The main aim of the paper was to identify conditions under which revision based on changes of the superiority relation was possible. Accordingly, the next important aspect of belief revision is to identify criteria of minimal change. It is possible to give alternative definitions of minimal revision. For example, one notion could be based on the cardinality of instances of the superiority relation, while another one is to consider minimality with respect to the conclusions derived from a theory. A few research questions naturally follow: 'Are there conditions on a theory to guarantee that a revision is minimal?', or 'Is it possible to compare different minimality criteria?'.

We illustrate some of these issue with the help of the following example.

Example 30. Consider $D=\left(\emptyset, R,\left\{\left(r_{10}, r_{6}\right)\right\}\right)$, with $R=\{$

$$
\begin{aligned}
& \Rightarrow_{r_{1}} a \Rightarrow_{r_{2}} \quad b \Rightarrow_{r_{3}} p \\
& \Rightarrow_{r_{4}} \neg a \Rightarrow_{r_{5}} \neg b \\
& \Rightarrow_{r_{6}} \quad c \quad \Rightarrow_{r_{7}} d \Rightarrow_{r_{8}} e \Rightarrow_{r_{9}} p \\
& \hat{\wedge}_{\left.r_{10} \neg c\right\} .}
\end{aligned}
$$


Consider now to change $>$ with

$$
\begin{aligned}
>^{\prime} & =\left\{\left(r_{6}, r_{10}\right)\right\} \\
>^{\prime \prime} & =\left\{\left(r_{1}, r_{4}\right),\left(r_{2}, r_{5}\right)\right\} .
\end{aligned}
$$

The superiority relation $>^{\prime}$ guarantees to change only two instances of the original superiority relation, but modifies the status of five literals $(c, \neg c, d, e$, and $p)$; on the contrary, $>^{\prime \prime}$ changes three instances, but only three literals $(a, b$ and $p)$ swap their status.

Lastly, the present work provides a further indication that the AGM postulates are not appropriate for belief revision of non-monotonic reasoning. Consequently, a natural question is whether there is a set of rational postulates for this kind of logics. We are sceptical about this endeavour: there are many different and often incompatible facets of non-monotonic reasoning, and a set of postulates might satisfy some particular nonmonotonic features but not being appropriate for others. For example, as we have seen in this paper, if we ignore monotonic conclusions (conclusions tagged with $\pm \Delta$ ), there are other cases where we cannot guarantee the success of the revision operation. On the other hand, [3] argues that the success postulate for revision holds if we are allowed to operate on rules instead of preferences. This example suggests that it might possible to find a set of postulates, but this would specific to a logic and specific types of operations. The quest for an alternative set of postulates for revision of non-monotonic theories is left for future research.

\section{Acknowledgements}

A previous version of the paper has been presented at the 13th International Workshop on Non-monotonic Reasoning (NMR 2010) [48] and to 4th International Web Rule Symposium (RuleML 2010) [49]. We thank the anonymous referees for NMR 2010 and RuleML 2010 for their valuable comments and feedback.

NICTA is funded by the Australian Government as represented by the Department of Broadband, Communications and the Digital Economy, the Australian Research Council through the ICT Centre of Excellence program and the Queensland Government.

\section{References}

1. Domshlak, C., Hüllermeier, E., Kaci, S., Prade, H.: Preferences in AI: An overview. Artificial Intelligence 175 (2011)

2. Katsuno, H., Mendelzon, A.O.: On the difference between updating a knowledge base and revising it. In Gärdenfors, P., ed.: Belief Revision. Cambridge University Press (1992) 183203

3. Billington, D., Antoniou, G., Governatori, G., Maher, M.J.: Revising non-monotonic belief sets: The case of defeasible logic. In: KI-99: Advances in Artificial Intelligence, SpringerVerlag (1999) 101-112 
4. Governatori, G., Rotolo, A.: Changing legal systems: Legal abrogations and annulments in defeasible logic. Logic Journal of the IGPL 18 (2010) 157-194

5. Delgrande, J.P., Schaub, T., Tompits, H., Woltran, S.: Belief revision of logic programs under answer set semantics. In Brewka, G., Lang, J., eds.: KR, AAAI Press (2008) 411-421

6. Delgrande, J.P.: A program-level approach to revising logic programs under the answer set semantics. TPLP 10 (2010) 565-580

7. Zhuang, Z., Delgrande, J.P., Nayak, A.C., Sattar, A.: Reconsidering AGM-style belief revision in the context of logic programs. In Kaminka, G.A., Fox, M., Bouquet, P., Hüllermeier, E., Dignum, V., Dignum, F., van Harmelen, F., eds.: ECAI 2016 - 22nd European Conference on Artificial Intelligence, 29 August-2 September 2016, The Hague, The Netherlands - Including Prestigious Applications of Artificial Intelligence (PAIS 2016). Volume 285 of Frontiers in Artificial Intelligence and Applications., IOS Press (2016) 671-679

8. Modgil, S.: Reasoning about preferences in argumentation frameworks. Artif. Intell. 173 (2009) 901-934

9. Moguillansky, M.O., Rotstein, N.D., Falappa, M.A., García, A.J., Simari, G.R.: Dynamics of knowledge in DeLP through argument theory change. TPLP 13 (2013) 893-957

10. Sartor, G.: Legal Reasoning. Springer, Dordrecht (2005)

11. Governatori, G., Olivieri, F., Scannapieco, S., Rotolo, A., Cristani, M.: Strategic argumentation is NP-complete. In Schaub, T., Friedrich, G., O'Sullivan, B., eds.: 21st European Conference on Artificial Intelligence (ECAI 2014). Volume 263 of Frontiers in Artificial Intelligence and Applications., IOS Press (2014) 399-404

12. Governatori, G., Maher, M.J., Billington, D., Antoniou, G.: Argumentation semantics for defeasible logics. Journal of Logic and Computation 14 (2004) 675-702

13. Antoniou, G., Maher, M.J., Billington, D.: Defeasible logic versus logic programming without negation as failure. J. Log. Program. 42 (2000) 47-57

14. Governatori, G.: On the relationship between Carneades and defeasible logic. In van Engers, T., ed.: Procedings of the 13th International Conference on Artificial Intelligence and Law (ICAIL 2011), ACM Press (2011)

15. Lam, H.P., Governatori, G., Riveret, R.: On ASPIC ${ }^{+}$and defeasible logic. In Baroni, P., Gordon, T.F., Scheffler, T., Stede, M., eds.: Proceedings of COMMA 2016. Volume 287 of Frontiers in Artificial Intelligence and Applications., Amsterdam, IOS Press (2016) 359-370

16. Antoniou, G., Billington, D., Governatori, G., Maher, M.J.: Representation results for defeasible logic. ACM Transactions on Computational Logic 2 (2001) 255-287

17. Antoniou, G., Billington, D., Governatori, G., Maher, M.J., Rock, A.: A family of defeasible reasoning logics and its implementation. In: ECAI. (2000) 459-463

18. Governatori, G., Padmanabhan, V., Rotolo, A., Sattar, A.: A defeasible logic for modelling policy-based intentions and motivational attitudes. Logic Journal of the IGPL 17 (2009) 227-265

19. Governatori, G., Rotolo, A., Olivieri, F., Scannapieco, S.: Legal contractions: a logical analysis. In Francesconi, E., Verheij, B., eds.: International Conference on Artificial Intelligence and Law, ICAIL '13, Rome, Italy, June 10-14, 2013, ACM (2013) 63-72

20. Antoniou, G., Billington, D., Governatori, G., Maher, M.J.: A flexible framework for defeasible logics. In: AAAI. (2000) 401-405

21. Billington, D., Antoniou, G., Governatori, G., Maher, M.J.: An inclusion theorem for defeasible logic. ACM Transactions in Computational Logic 12 (2010) article 6

22. Antoniou, G., Billington, D., Governatori, G., Maher, M.J.: Embedding defeasible logic into logic programming. TPLP 6 (2006) 703-735

23. Maher, M.J.: Propositional defeasible logic has linear complexity. Theory and Practice of Logic Programming 1 (2001) 691-711

24. Antoniou, G., Billington, D., Governatori, G., Maher, M.J.: Embedding defeasible logic into logic programming. Theory and Practice of Logic Programming 6 (2006) 703-735 
25. Alchourrón, C.E., Gärdenfors, P., Makinson, D.: On the logic of theory change: Partial meet contraction and revision functions. J. Symb. Log. 50 (1985) 510-530

26. Levi, I.: Subjunctives, dispositions, and chances. Synthese 34 (1977) 423-455

27. Gärdenfors, P.: Knowledge in Flux: Modeling the Dynamics of Epistemic States. Cambridge (1988)

28. Inoue, K., Sakama, C.: Abducing priorities to derive intended conclusions. In Dean, T., ed.: IJCAI, Morgan Kaufmann (1999) 44-49

29. Boella, G., Governatori, G., Rotolo, A., van der Torre, L.: Lex minus dixit quam voluit, lex magis dixit quam voluit: A formal study on legal compliance and interpretation. In: AI approaches to the complexity of legal systems, Berlin, Springer (2010)

30. Boella, G., Governatori, G., Rotolo, A., van der Torre, L.: A logical understanding of legal interpretation. In: Proceedings of KR 2010. (2010)

31. Dung, P.M.: An argumentation semantics for logic programming with explicit negation. In: ICLP. (1993) 616-630

32. Dung, P.M.: On the acceptability of arguments and its fundamental role in nonmonotonic reasoning, logic programming and n-person games. Artif. Intell. 77 (1995) 321-358

33. Moguillansky, M.O., Rotstein, N.D., Falappa, M.A., García, A.J., Simari, G.R.: Argument theory change applied to defeasible logic programming. In Fox, D., Gomes, C.P., eds.: AAAI, AAAI Press (2008) 132-137

34. Moguillansky, M.O., Rotstein, N.D., Falappa, M.A., García, A.J., Simari, G.R.: Argument theory change through defeater activation. In Baroni, P., Cerutti, F., Giacomin, M., Simari, G.R., eds.: COMMA. Volume 216 of Frontiers in Artificial Intelligence and Applications., IOS Press (2010) 359-366

35. Prakken, H., Sartor, G.: Argument-based extended logic programming with defeasible priorities. Journal of Applied Non-Classical Logics 7 (1997)

36. Antoniou, G.: Defeasible logic with dynamic priorities. Int. J. Intell. Syst. 19 (2004) 463-472

37. Brewka, G.: Well-founded semantics for extended logic programs with dynamic preferences. Journal of Artificial Intelligence Research 4 (1996) 19-36

38. Modgil, S.: Hierarchical argumentation. In Fisher, M., van der Hoek, W., Konev, B., Lisitsa, A., eds.: JELIA. Volume 4160 of Lecture Notes in Computer Science., Springer (2006) 319332

39. Nute, D.: Defeasible logic. In: Handbook of Logic in Artificial Intelligence and Logic Programming. Volume 3. Oxford University Press (1994) 353-395

40. Bassiliades, N., Antoniou, G., Vlahavas, I.: A defeasible logic reasoner for the semantic web. International Journal of Semantic Web and Information Systems 2 (2006) 1-41

41. Antoniou, G., Bikakis, A.: DR-Prolog: A system for defeasible reasoning with rules and ontologies on the semantic web. IEEE Transaction on Knowledge and Data Engineering 19 (2007) 233-245

42. Lam, H.P., Governatori, G.: The making of SPINdle. In Paschke, A., Governatori, G., Hall, J., eds.: Proceedings of The International RuleML Symposium on Rule Interchange and Applications (RuleML 2009), Springer (2009) 315-322

43. Governatori, G., Olivieri, F., Rotolo, A., Scannapieco, S., Sartor, G.: Two faces of strategic argumentation in the law. In Hoekstra, R., ed.: The Twenty-Seventh Annual Conference on Legal Knowledge and Information Systems (JURIX 2014). Volume 271 of Frontiers in Artificial Intelligence and Applications., IOS Press (2014) 81-90

44. Maher, M.J.: Complexity of exploiting privacy violations in strategic argumentation. In Pham, D.N., Park, S., eds.: 13th Pacific Rim International Conference on Artificial Intelligence (PRICAI 2014). Volume 8862 of Lecture Notes in Computer Science., Springer (2014) 523-535

45. Cristani, M.: Many-sorted preference relations. In Fensel, D., Giunchiglia, F., McGuinness, D.L., Williams, M.A., eds.: KR, Morgan Kaufmann (2002) 265-276 
46. Düntsch, I.: A microcomputer based system for small relation algebras. J. Symb. Comput. 18 (1994) 83-86

47. Cristani, M., Hirsch, R.: The complexity of constraint satisfaction problems for small relation algebras. Artif. Intell. 156 (2004) 177-196

48. Governatori, G., Olivieri, F., Scannapieco, S., Cristani, M.: Superiority based revision of defeasible theories. In Meyer, T., Ternovska, E., eds.: 13 International Workshop on NonMonotonic Reasoning (NMR 2010). (2010)

49. Governatori, G., Olivieri, F., Scannapieco, S., Cristani, M.: Superiority based revision of defeasible theories. In Dean, M., Hall, J., Rotolo, A., Tabet, S., eds.: RuleML 2010: 4th International Web Rule Symposium. Number 6403 in LNCS, Berlin, Springer (2010) 104118 